\title{
Strong lensing in Abell 1703: constraints on the slope of the inner dark matter distribution ${ }^{\star}$
}

\author{
M. Limousin ${ }^{1,2}$, J. Richard ${ }^{3}$, J.-P. Kneib ${ }^{4}$, H. Brink ${ }^{2}$, R. Pelló ${ }^{5}$, E. Jullo ${ }^{4}$, H. Tu ${ }^{6,7,8}$, J. Sommer-Larsen ${ }^{9,2}$, \\ E. Egami ${ }^{10}$, M. J. Michałowski ${ }^{2}$, R. Cabanac ${ }^{1}$, and D. P. Stark ${ }^{3}$
}

${ }^{1}$ Laboratoire d'Astrophysique de Toulouse-Tarbes, Université de Toulouse, CNRS, 57 avenue d'Azereix, 65000 Tarbes, France e-mail: marceau. limousin@ast.obs-mip. fr

2 Dark Cosmology Centre, Niels Bohr Institute, University of Copenhagen, Juliane Maries Vej 30, 2100 Copenhagen, Denmark

3 Department of Astronomy, California Institute of Technology, 105-24, Pasadena, CA91125, USA

${ }^{4}$ Laboratoire d'Astrophysique de Marseille, CNRS-Université de Provence, 38 rue Frédéric Joliot-Curie, 13388 Marseille Cedex 13 , France

${ }^{5}$ Laboratoire d'Astrophysique de Toulouse-Tarbes, Université de Toulouse, CNRS, 14 avenue Edouard Belin, 31400 Toulouse, France

6 Physics Department, Shanghai Normal University, 100 Guilin Road, Shanghai 200234, PR China

7 Institut d'Astrophysique de Paris, CNRS, 98bis Bvd Arago, 75014 Paris, France

8 Shanghai Astronomical Observatory, 80 Nandan Road, Shanghai 200030, PR China

9 Excellence Cluster Universe, Technische Universität München, Boltz-manstr. 2, 85748 Garching, Germany

10 Steward Observatory, University of Arizona, 933 North Cherry Avenue, Tucson, AZ 85721, USA

Received 25 February 2008 / Accepted 12 July 2008

\section{ABSTRACT}

\begin{abstract}
Properties of dark matter haloes can be probed observationally and numerically, and comparing both approaches provides ways to constrain cosmological models. When it comes to the inner part of galaxy cluster scale haloes, interaction between the baryonic and the dark matter component is an important issue that is far from being fully understood. With this work, we aim to initiate a program coupling observational and numerical studies to probe the inner part of galaxy clusters. In this article, we apply strong lensing techniques on Abell 1703, a massive X-ray luminous galaxy cluster at $z=0.28$. Our analysis is based on imaging data from both the space and ground in 8 bands, complemented by a spectroscopic survey. Abell 1703 is rather circular from the general shape of its multiply imaged systems and is dominated by a giant elliptical cD galaxy in its centre. This cluster exhibits a remarkable bright central ring formed by 4 images at $z_{\text {spec }}=0.888$ only $5-13^{\prime \prime}$ away from the cD centre. This unique feature offers a rare lensing constrain for probing the central mass distribution. The stellar contribution from the cD galaxy $\left(\sim 1.25 \times 10^{12} M_{\odot}\right.$ within $\left.30 \mathrm{kpc}\right)$ is accounted for in our parametric mass modelling, and the underlying smooth dark matter component distribution is described using a generalized NFW profile parametrized with a central logarithmic slope $\alpha$. The rms of our mass model in the image plane is equal to $1.4^{\prime \prime}$. We find that within the range where observational constraints are present (from $\sim 20 \mathrm{kpc}$ to $\sim 210 \mathrm{kpc}$ ), $\alpha$ is equal to $1.09_{-0.11}^{+0.05}$ ( $3 \sigma$ confidence level). The concentration parameter is equal to $c_{200} \sim 3.5$, and the scale radius is constrained to be larger than the region where observational constraints are available $\left(r_{\mathrm{s}}=730_{-75}^{+15} \mathrm{kpc}\right)$. The $2 \mathrm{D}$ mass is equal to $M(210 \mathrm{kpc})=2.4 \times 10^{14} M_{\odot}$. However, we cannot draw any conclusions on cosmological models at this point since we lack results from realistic numerical simulations containing baryons to make a proper comparison. We advocate the need for a large sample of well observed (and well constrained) and simulated unimodal relaxed galaxy clusters in order to make reliable comparisons and to potentially provide a test of cosmological models.
\end{abstract}

Key words. gravitational lensing - galaxies: clusters: individual: Abell 1703 - dark matter

\section{Introduction}

Large $N$-body cosmological simulations have been carried out for a decade, with the goal of making statistical predictions on dark matter (DM) halo properties. Because of numerical issues, most of these large cosmological simulations contain dark matter particles only. They all reliably predict that the 3D density profile $\rho_{\mathrm{DM}}(r)$ should fall as $r^{-3}$ beyond what is usually called the scale radius. Observations have confirmed these predictions (e.g. Kneib et al. 2003; Pointecouteau et al. 2005; Mandelbaum et al. 2008). This agreement is likely to be connected with the fact that at large radius, the density profile of a galaxy cluster is dark matter dominated and the influence of baryons can be

\footnotetext{
* Appendix A is only available in electronic form at http://www . aanda.org
}

neglected. On smaller scales, if we parametrize the 3D density profile of the DM using a cuspy profile $\rho_{\mathrm{DM}} \propto r^{-\alpha}$; dark matter only simulations predict a logarithmic slope $\alpha \sim 1-1.5$ for $r \rightarrow 0$. The exact value of the central slope and its universality is debated (Navarro et al. 1997; Moore et al. 1998; Ghigna et al. 2000; Ricotti 2003; Navarro et al. 2004; Gao et al. 2008). From the theoretical point of view, the logarithmic slope of DM haloes is predicted to be $\alpha \sim 0.8$ (Austin et al. 2005; Hansen \& Stadel 2006). Although this debate is of some interest, these dark matter only studies and their predictions do not help much to make comparison with observations. Indeed, observing the central part (i.e. the inner $\sim 500 \mathrm{kpc}$ ) of a galaxy cluster at any wavelength reveals the presence of baryons (in the forms of stars and X-ray hot gas). Thus any attempt to compare observations to simulations in the centre of galaxy clusters has to be made with 
numerical simulations (or calculations) taking into account the baryonic component and its associated physics.

Efforts are currently developed on the numerical side in order to account for the presence of baryons (e.g. the Horizon ${ }^{1}$ simulation). In practice, our understanding of the baryonic physics is poor, and the exact interplay between dark matter and baryon is far from understood. For example, due to the overcooling problem, numerical simulations predict blue central brightest cluster galaxies which are not always observed. Different effects do compete when it comes to the central slope of the density profile: the cooling of gas in the centre of dark matter haloes is expected to lead to a more concentrated dark matter distribution (the so-called adiabatic contraction, see Blumenthal et al. 1986; Gnedin et al. 2004; Gustafsson et al. 2006). On the other hand, dynamical friction heating of massive galaxies against the diffuse cluster dark matter can flatten the slope of the DM density profile (El-Zant et al. 2001; Nipoti et al. 2003, 2004; Ma \& Boylan-Kolchin 2004), and this effect could even dominate over adiabatic contraction (El-Zant et al. 2004). Note also that the properties of the inner part of simulated galaxy clusters (even in dark matter only simulations) can depend significantly on initial conditions as demonstrated in Kazantzidis et al. (2004). To summarize, no coherent picture has yet emerged from $N$-body simulations when it comes to the shape of the inner density profile of structures; this problem is a difficult one and the answer is likely not to be unique but may depend on the physical properties of the structures and their formation history.

On the observational side, efforts have been put on probing the central slope $\alpha$ of the underlying dark matter distribution. These analyses have led to wide-ranging results, whatever the method used: X-ray (Ettori et al. 2002; Arabadjis et al. 2002; Lewis et al. 2003; Zappacosta et al. 2006); lensing (Tyson et al. 1998; Smith et al. 2001; Dahle et al. 2003; Sand et al. 2002; Gavazzi et al. 2003; Gavazzi 2005; Sand et al. 2004, 2008; Bradač et al. 2007) or dynamics (Kelson et al. 2002; Biviano \& Salucci 2006). This highlight the difficulty of such studies and the possible large scatter in the value of $\alpha$ from one cluster to another.

To summarize, we need to probe observationally and numerically the behaviour of the underlying dark matter distribution (i.e. after the baryonic component has been separated from the dark matter component) in the central parts of galaxy clusters. The main difficulties are: i) observationally, to be able to disentangle the baryonic component and the underlying dark matter distribution; ii) numerically, to implement the baryonic physics into the simulations; iii) then to compare both approaches in a consistent way. These issues are far beyond the scope of this article, but they are likely to provide an interesting test of the $\Lambda \mathrm{CDM}$ scenario in the future.

In this work, we aim to probe observationally the central (i.e. from $\sim 5^{\prime \prime}(\sim 20 \mathrm{kpc})$ up to $\sim 50^{\prime \prime}(\sim 200 \mathrm{kpc})$ from the centre) density profile of a massive cluster lens. Our main goal is to measure the slope of the inner underlying dark matter distribution within this radius. In practice, we apply strong lensing techniques on galaxy cluster Abell 1703, a massive $z=0.28$ (Allen et al. 1992) X-ray cluster with a luminosity $L_{\mathrm{x}}=8.66 \times 10^{44} \mathrm{erg} \mathrm{s}^{-1}$ (Böhringer et al. 2000). It is very well suited for the analysis we want to perform for the following reasons:

1. it contains a large number of gravitational arcs, providing many lensing constraints for the analysis (Fig. 1);

${ }^{1}$ http://www . projet-horizon. fr
2. although it displays an intriguing filamentary structure along the north-south direction, it looks rather circular from the geometrical configuration of its multiply imaged systems, in particular its giant arc, located at large angular separation $\left(\sim 35^{\prime \prime} \sim 147 \mathrm{kpc}\right)$. It is likely to be a unimodal cluster, with a clear dominant elliptical cD galaxy, which makes it much easier to interpret the results of the modelling compared to bimodal clusters such as Abell 1689 (Limousin et al. 2007b), Abell 2218 (Elíasdóttir et al. 2007), Abell 68 (Richard et al. 2007) or MS 2053.7-0449 (Verdugo et al. 2007). Indeed, regular relaxed clusters are rare at such redshifts (Smith et al. 2005);

3. it presents a remarkable lensing configuration, forming a central ring composed of four bright images (see Fig. 1 and Appendix). Interestingly, these constraints are found very close to the cD galaxy, potentially providing robust constraints in the very central part of the cluster. Note that this central ring is not really central since it does not coincide with the peak of the total mass distribution.

This article is organized as follows: data used in this work are presented in Sect. 2. Section 3 presents the multiply imaged systems that constitutes the basis of our analysis. The strong lensing analysis is outlined in Sect. 4, and the results are given in Sect. 5. We discuss our results and conclude in Sect. 6.

All our results are scaled to the flat, $\Lambda$ CDM cosmology with $\Omega_{\mathrm{M}}=0.3, \Omega_{\Lambda}=0.7$ and a Hubble constant $\mathrm{H}_{0}=70 \mathrm{~km} \mathrm{~s}^{-1} \mathrm{Mpc}^{-1}$. In such a cosmology, at $z=0.28$, $1^{\prime \prime}$ corresponds to $4.244 \mathrm{kpc}$. All the figures of the cluster are aligned with WCS coordinates, i.e. north is up, east is left. The reference centre of our analysis is fixed at the $\mathrm{cD}$ centre: $\mathrm{RA}=$ 13:15:05.276, Dec $=+51: 49: 02.85$ (J 2000.0). Magnitudes are given in the $\mathrm{AB}$ system.

\section{Data}

Abell 1703 has been observed from space by the Hubble Space Telescope (using ACS and NICMOS); from ground with the Subaru telescope (using the Multi-Object InfraRed Camera and Spectrograph, MOIRCS, Ichikawa et al. 2006) and with the Keck telescope (using the Low Resolution Imaging Spectrograph, LRIS, Oke et al. 1995). Table 2 sums up the different dataset.

\subsection{Imaging data}

The multiwavelength ACS data have been used to construct colour images of Abell 1703 in order to identify the multiply imaged systems. In addition to the 6 ACS bands, we benefited from NICMOS and Subaru data in order to construct spectral energy distributions (SED) and estimate photometric redshifts for the multiple images as well as the stellar mass of the cD galaxy.

HST/ACS: we use the HST ACS data in six bands. Abell 1703 has been observed on May 2005 as part of the ACS team guaranteed observing time (P.I. Holland Ford, proposal 10325). These images have been reduced using the multidrizzle software to remove cosmic rays, bad pixels, combine the dithered frames and correct for geometric distortions. The output pixel scale was fixed at 0.04 " and we used a pixfrac parameter value of 0.8 for reducing the area of the input pixels. 


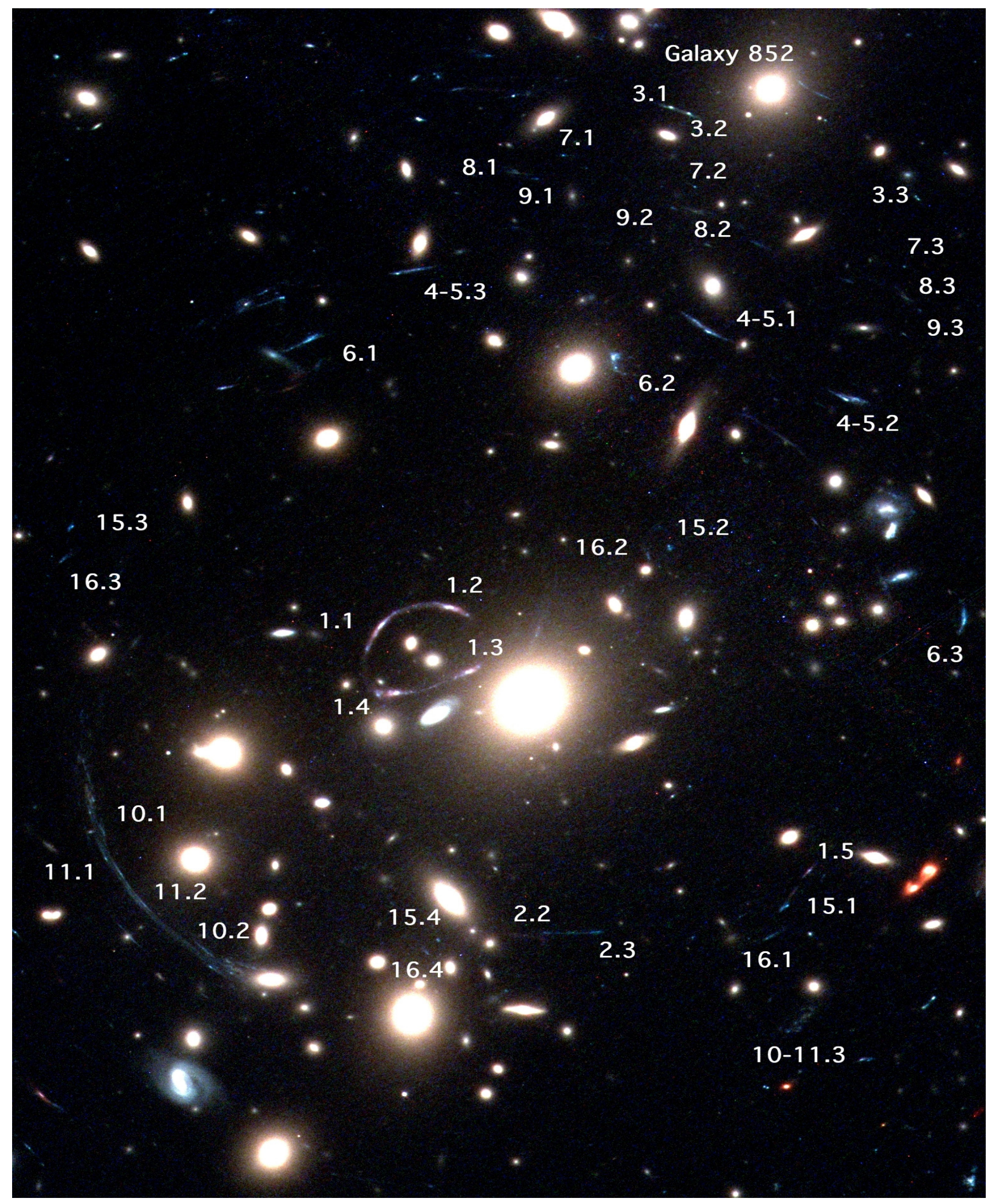

Fig. 1. Colour image of Abell 1703 from F850W, F625W and F475W observations (Stott 2007). North is up, east is left. Size of the field of view is equal to $77^{\prime \prime} \times 107^{\prime \prime}$, corresponding to $326 \mathrm{kpc} \times 454 \mathrm{kpc}$. Multiply imaged systems used in the analysis are shown, and colour images are given in Appendix. The central ring formed by four bright images is found close to the cD galaxy. The giant arc (systems 10-11) falls south-east, at distance of $\sim 35^{\prime \prime}$. System 2 is a straight arc located south of the cD and composed of two merging images. System 15 and 16 follow each other, forming a kind of Einstein cross configuration. In the north, we find a set of tangential systems (4-5-6-7-8-9). Then, two bright merging images form system 3 , located close to galaxy 852 which present a blue nearby lensing feature. The filamentary structure can be appreciated on this image. See also the Subaru $H$ band image in Appendix which is more extended. 

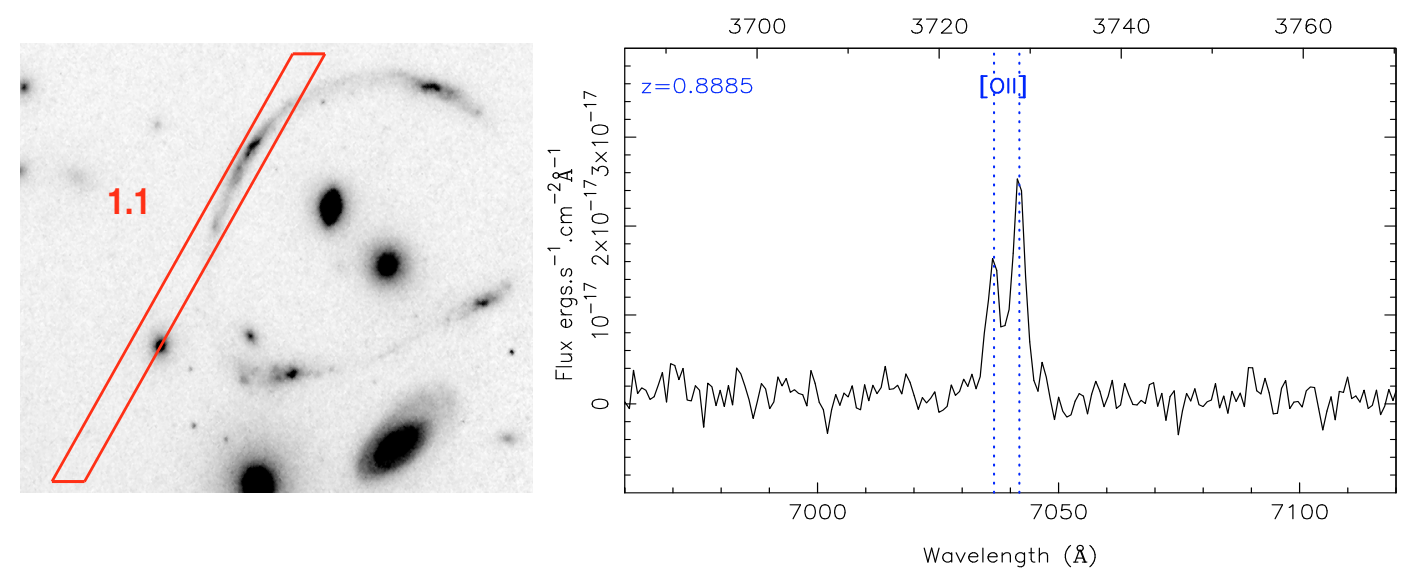

Fig. 2. Spectroscopic observation of image 1.1. Left: position of the slit. Right: $1 \mathrm{D}$ spectrum. The $\left[\mathrm{O}_{\mathrm{II}}\right]_{3726,3729}$ doublet is easily identified.

HST/NICMOS: Abell 1703 has been observed with NICMOS on October 10, 2006 (P.I. Holland Ford, proposal 10996), using the $F 110 \mathrm{~W}$ filter. NICMOS images have been reduced following the NICMOS data reduction handbook ${ }^{2}$ and including specific improvements to remove cosmic rays, quadrant-toquadrant variations, bias and flat residuals. More details on these improvements are given in Richard et al. (2008).

Subaru MOIRCS: MOIRCS observations of Abell 1703 were obtained in May 2007 (PI: Egami). We took five series of dithered exposures, with individual exposure times of 20, 25 and $30 \mathrm{~s}$ and 5 coadds per frame, producing a total exposure time of $18875 \mathrm{~s}$ in the central region. These observations were obtained under excellent seeing conditions $\left(0.3-0.4^{\prime \prime}\right)$. We used the MCSRED package ${ }^{3}$ to perform flat-fielding, sky subtraction, distortion correction and mosaicking of individual images. The photometric calibration was derived with the observed magnitudes of 15 stars from the 2MASS catalogue.

\subsection{Spectroscopic data}

We used LRIS on Keck I in an attempt to measure a redshift for the brightest component of system 1 (image 1.1). Four exposures of $900 \mathrm{~s}$ were taken on Jan. 29th 2008, under photometric conditions but a poor seeing $\left(1.4^{\prime \prime}\right)$, using the 900 lines $\mathrm{mm}^{-1}$ grating blazed at $6320 \AA$ in the red channel of the instrument. This setup covers the wavelength range 5600-7200 $\AA$ at a resolution of $2.77 \AA$ and a dispersion of $0.83 \AA$ per pixel. The spectrum has been reduced with standard IRAF procedures for bias correction, flat-fielding, sky subtraction and distortion correction, in that order. We used the numerous sky lines in this region for the wavelength calibration and observation of the standard star Feige 92 for the flux calibration.

We detected a bright doublet of emission lines, centred at 7036 and $7041 \AA$ respectively, which we interpret as $\left[\mathrm{O}_{\mathrm{II}}\right]_{3726,3729}$ at $z=0.8885 \pm 0.0002$ without any ambiguity, the doublet being easily separated by $5 \AA$ at this resolution (Fig. 2). The spectroscopic redshift is in agreement with our photometric redshift estimate of $z_{\text {phot }}=0.965_{-0.240}^{+0.075}$ (Table 1).

\footnotetext{
2 http://wWw. stsci.edu/hst/nicmos/documents/handbooks

${ }^{3}$ http://www .naoj.org/staff/ichi/MCSRED/mcsred.html
}

\subsection{Photometric analysis}

We created photometric catalogues combining the multicolour images by running SExtractor (Bertin \& Arnouts 1996) in double image mode. A specific detection image was created by combining all the ACS data after scaling them to the same background noise level. We used the $\mathrm{cD}$-subtracted images (see Sect. 2.6) for ACS and MOIRCS to prevent any strong photometric contamination in the central regions, in particular for the measurement of the lensed ring-shape system (system 1). Photometry was optimized to the small size of the lensed background galaxies, by measuring the flux in a $1.0^{\prime \prime}$ diameter aperture. This gives accurate colours in the optical bands that used the same instrument. We estimated aperture corrections in the near-infrared by measuring the photometry of 10 isolated bright point sources in the NICMOS and MOIRCS images, and corrected our photometric catalogues for this difference.

We increased the photometric error bars, usually underestimated by SExtractor, to take into account the effects of drizzling in the reduction of ACS and NICMOS images, following the computations by Casertano et al. (2000). For the MOIRCS images, we measured the pixel-to-pixel background noise from blank regions of sky selected in the original images, and scaled it to the aperture size used in the photometry.

\subsection{Cluster member identification}

To extract cluster galaxies, we plot the characteristic cluster red sequences $(\mathrm{F} 775 \mathrm{~W}-\mathrm{H})$ and $(\mathrm{F} 625 \mathrm{~W}-\mathrm{H})$ in two colourmagnitude diagrams and select the objects lying on both redsequences as cluster galaxies. This yields 345 early-type cluster galaxies down to $F 775 W=24$. For the purpose of the modelling, we will consider only the cluster members whose magnitude is brighter than 21 in the $F 775 \mathrm{~W}$ band (in order to save computing time, see Elíasdóttir et al. 2007) and which are located close to some multiply imaged systems. We consider fainter galaxies only if they are located close to some multiple images since they can locally perturb the lensing configuration (Meneghetti et al. 2007a). This yields 45 galaxy scale perturbers.

\subsection{Photometric redshifts}

We ran the photometric redshift code HyperZ (Bolzonella et al. 2000) on the multi-band photometric catalogues, in order to get a redshift estimate for all the multiple images identified in the field. This program performs a minimization procedure 
Table 1. Multiply imaged systems considered in this work.

\begin{tabular}{|c|c|c|c|c|c|c|c|c|}
\hline Id & RA & Dec & $z_{\text {phot }}$ & $\Delta\left(z_{\text {phot }}\right)$ & $z_{\mathrm{mod}}$ & $\mathrm{rms}(s)$ & rms $(i)$ & $z_{\text {free }}$ \\
\hline 1.1 & 198.77725 & 51.81934 & $0.965_{-0.240}^{+0.075}$ & $-0.8885-$ & - & 0.27 & 1.95 & \\
\hline 1.2 & 198.77482 & 51.81978 & $0.940_{-0.096}^{+0.102}$ & $\ldots$ & & & & \\
\hline 1.3 & 198.77414 & 51.81819 & $0.995_{-0.087}^{+0.051}$ & . & & & & \\
\hline 1.4 & 198.77671 & 51.81767 & $0.740_{-0.078}^{+0.129}$ & $\ldots$ & & & & \\
\hline 1.5 & 198.76206 & 51.81331 & $0.915_{-0.090}^{+0.108}$ & $\ldots$ & & & & \\
\hline 2.2 & 198.77156 & 51.81174 & $2.310_{-0.381}^{+0.456}$ & [1.9-2.8] & $2.24_{-0.14}^{+0.25}$ & 0.09 & 0.39 & $3.98_{-0.52}^{+1.30}$ \\
\hline 2.3 & 198.76970 & 51.81186 & $2.225_{-0.657}^{+0.195}$ & $\ldots$ & & & & \\
\hline 3.1 & 198.76696 & 51.83205 & - & [3.31-3.37] & $3.32_{-0.01}^{+0.03}$ & 0.15 & 1.22 & $4.85_{-0.71}^{+0.65}$ \\
\hline 3.2 & 198.76634 & 51.83190 & $3.350_{-0.036}^{+0.024}$ & $\ldots$ & & & & \\
\hline 3.3 & 198.75824 & 51.82982 & $3.350_{-0.036}^{+0.030}$ & $\ldots$ & & & & \\
\hline 4.1 & 198.76564 & 51.82653 & - & {$[2.03-2.48]$} & $2.03_{-0.00}^{+0.03}$ & 0.17 & 0.51 & $2.46_{-0.11}^{+0.12}$ \\
\hline 4.2 & 198.76075 & 51.82487 & $2.255_{-0.222}^{+0.216}$ & $\ldots$ & & & & \\
\hline 4.3 & 198.77666 & 51.82797 & - & $\ldots$ & & & & \\
\hline 5.1 & 198.76602 & 51.82677 & - & [2.03-2.48] & $2.03_{-0.00}^{+0.03}$ & 0.21 & 0.64 & $2.36_{-0.08}^{+0.14}$ \\
\hline 5.2 & 198.76041 & 51.82489 & $2.315_{-0.186}^{+0.171}$ & $\ldots$ & & & & \\
\hline 5.3 & 198.77591 & 51.82807 & - & $\ldots$ & & & & \\
\hline 6.1 & 198.77984 & 51.82640 & $2.595_{-0.159}^{+0.144}$ & {$[2.43-2.79]$} & $2.78_{-0.06}^{+0.00}$ & 0.30 & 0.84 & $4.98_{-0.49}^{+0.45}$ \\
\hline 6.2 & 198.76890 & 51.82580 & $2.535_{-0.117}^{+0.258}$ & $\ldots$ & & & & \\
\hline 6.3 & 198.75652 & 51.81947 & $2.625_{-0.135}^{+0.1108}$ & $\ldots$ & & & & \\
\hline 7.1 & 198.77074 & 51.83087 & $3.490_{-0.108}^{+0.133}$ & {$[2.52-3.62]$} & $3.59_{-0.18}^{+0.02}$ & 0.28 & 2.59 & $5.63_{-0.56}^{+0.02}$ \\
\hline 7.2 & 198.76614 & 51.83010 & $2.960_{-0.183}^{+0.354}$ & $\ldots$ & & & & \\
\hline 7.3 & 198.75869 & 51.82814 & $3.200_{-0.675}^{+0.210}$ & $\ldots$ & & & & \\
\hline 8.1 & 198.77250 & 51.83045 & $2.805_{-0.090}^{+0.017}$ & [2.61-2.98] & $2.97_{-0.05}^{+0.00}$ & 0.30 & 1.63 & $5.53_{-0.61}^{+0.08}$ \\
\hline 8.2 & 198.76608 & 51.82949 & $2.770_{-0.108}^{+0.207}$ & $\ldots$ & & & & \\
\hline 8.3 & 198.75863 & 51.82740 & $2.725_{-0.117}^{+0.198}$ & $\ldots$ & & & & \\
\hline 9.1 & 198.77176 & 51.83030 & - & {$[2.40-3.37]$} & $3.36_{-0.07}^{+0.04}$ & 0.24 & 1.29 & $5.53_{-0.61}^{+0.08}$ \\
\hline 9.2 & 198.76690 & 51.82957 & $2.995_{-0.378}^{+0.195}$ & $\ldots$ & & & & \\
\hline 9.3 & 198.75813 & 51.82708 & $3.000_{-0.603}^{+0.366}$ & $\ldots$ & & & & \\
\hline 10.1 & 198.78708 & 51.81424 & $3.100_{-0.162}^{+0.324}$ & {$[2.40-3.42]$} & $2.41_{-0.01}^{+0.07}$ & 0.21 & 1.62 & $3.79_{-0.28}^{+0.25}$ \\
\hline 10.2 & 198.78352 & 51.81138 & $2.595_{-0.189}^{+0.117}$ & $\ldots$ & & & & \\
\hline 10.3 & 198.76242 & 51.80954 & $2.705_{-0.261}^{+0.189}$ & $\ldots$ & & & & \\
\hline 11.1 & 198.78648 & 51.81322 & $3.045_{-0.138}^{+0.102}$ & {$[2.40-3.42]$} & $2.47_{-0.04}^{+0.06}$ & 0.25 & 2.13 & $3.79_{-0.27}^{+0.27}$ \\
\hline 11.2 & 198.78564 & 51.81247 & $3.155_{-0.099}^{+0.078}$ & $\ldots$ & & & & \\
\hline 11.3 & 198.76242 & 51.80954 & $2.705_{-0.261}^{+0.189}$ & $\ldots$ & & & & \\
\hline 15.1 & 198.76284 & 51.81246 & $2.440_{-0.231}^{+0.171}$ & [2.21-2.67] & $2.67_{-0.09}^{+0.00}$ & 0.37 & 0.93 & $5.58_{-0.71}^{+0.26}$ \\
\hline 15.2 & 198.76704 & 51.82128 & $2.440_{-0.153}^{+0.231}$ & $\cdots$ & & & & \\
\hline 15.3 & 198.78821 & 51.82176 & - & $\ldots$ & & & & \\
\hline 15.4 & 198.77519 & 51.81155 & - & $\ldots$ & & & & \\
\hline 16.1 & 198.76356 & 51.81164 & $2.710_{-0.324}^{+0.216}$ & {$[2.50-2.92]$} & $2.70_{-0.08}^{+0.10}$ & 0.27 & 0.73 & $5.04_{-0.52}^{+0.44}$ \\
\hline 16.2 & 198.76774 & 51.82101 & - & $\ldots$ & & & & \\
\hline 16.3 & 198.78838 & 51.82097 & $2.750_{-0.165}^{+0.171}$ & $\ldots$ & & & & \\
\hline 16.4 & 198.77558 & 51.81128 & - & $\ldots$ & & & & \\
\hline
\end{tabular}

We have found 13 distinct multiply imaged systems. Coordinates are given in degrees (J 2000.0). When the photometry is reliable in each band, we report the corresponding photometric redshift estimates, with error bars quoting the $3 \sigma$ confidence level. $\Delta\left(z_{\text {phot }}\right)$ corresponds to the redshift range allowed by the photometric redshift estimation and that will be used as a prior in the optimization (i.e. for each system, the redshift will be let free and allowed to vary between $\left.\Delta\left(z_{\text {phot }}\right)\right)$. For system 1 however we fix the redshift to the measured one, 0.8885 . The photometric estimate $\Delta\left(z_{\text {phot }}\right)=[0.725-1.04]$ is in agreement with the spectroscopic measurement. $z_{\bmod }$ corresponds to the redshift inferred from the optimization procedure. We report both the rms in the source plane and the rms in the image plane. The mean scatters are given for the whole system, not for each individual image composing a system. The total rms is equal to $0.26^{\prime \prime}$ (source plane) and $1.45^{\prime \prime}$ (image plane). $z_{\text {free }}$ corresponds to the redshift inferred from the optimization when all redshift but system 1 are assigned a flat prior between 0.28 and 6 (see Sect. 5.3).

between the spectral energy distribution of each object and a library of spectral templates, either empirical (Kinney et al. 1996; Coleman et al. 1980) or from the evolutionary models by
Bruzual \& Charlot (2003). Note that the NICMOS data we have correspond to a mosaic of four pointings, none of them being centred on the $\mathrm{cD}$ galaxy. It results that the $\mathrm{cD}$ galaxy is not 
Table 2. Different observations of Abell 1703 used in this work: instrument, filter and exposure time in seconds.

\begin{tabular}{ccc}
\hline \hline Instrument & Filter & Exp. time (s) \\
\hline ACS & $F 435 W$ & 7050 \\
ACS & $F 475 W$ & 5564 \\
ACS & $F 555 W$ & 5564 \\
ACS & $F 625 W$ & 8494 \\
ACS & $F 775 W$ & 11128 \\
ACS & $F 850 W$ & 17800 \\
NICMOS & $F 110 W$ & 2624 \\
MOIRCS & $H$ & 18875 \\
\hline
\end{tabular}

fully sampled by the NICMOS data. Therefore, it cannot be subtracted in the NICMOS image, thus we did not take into account the $F 110 \mathrm{~W}$ filter measurement for all its neighbouring objects, or for multiple images located at the edges of the NICMOS field of view. Absolute photometric calibration between the different bands is usually accurate down to $0.05 \mathrm{mag}$, we used this value as a minimal value in the code (this value will be used also for fitting the SED of the cD galaxy in Sect. 2.6). We scanned the following range of parameters: $0.0<z<7.0$ for the redshift, $0.0<A_{\mathrm{V}}<1.2$ for the reddening, applied on the template spectra with the Calzetti et al. (2000) law observed in starburst galaxies. Optical depth in the Lyman- $\alpha$ forest followed the Madau (1995) prescription.

The results obtained for each multiple image are reported in Table 1, along with the $3 \sigma$ error bar estimate given by HyperZ from the redshift probability distribution.

\section{6. $c D$ galaxy}

Abell 1703 exhibits a dominant central giant elliptical galaxy: its stellar contribution to the mass budget in the central part is to be taken properly into account. We worked out some of the $\mathrm{cD}$ galaxy properties from the broad band photometry. In particular, we are interested in computing its luminosities in the different filters in order to estimate its stellar mass. Note again that we have not been able to study the $\mathrm{cD}$ galaxy in the NICMOS band since the NICMOS data correspond to a mosaic of four pointings, none of them being centred on the $\mathrm{cD}$ galaxy.

Subtraction of the cD galaxy: we fitted and subtracted from each image a model representation of the surface brightness distribution using the IRAF task ellipse. Both the position angle and ellipticity were allowed to vary as a function of the semimajor axis in the fitted elliptical isophotes, as well as the isophote centroid in the central part. This procedure was found to give satisfactory residuals at the centre (see Appendix). This procedure also allows us to determine accurate photometry for the $\mathrm{cD}$ galaxy, that we report in Table 3. The integrated luminosity profile in the rest frame $B$ band is shown in Fig. 3, together with a mass profile of the $\mathrm{cD}$ galaxy as included in the modelling (see Sect. 4.2).

Stellar mass: from the broad band photometry, we estimate the stellar mass of the $\mathrm{cD}$ galaxy by fitting its spectral energy distribution using the HyperZ software. In order to perform this estimation, we choose an aperture equal to $7^{\prime \prime}$. This choice is
Table 3. Luminosities and stellar mass to light ratio computed in different rest frame filters.

\begin{tabular}{ccccc}
\hline \hline Filter & $L($ no Av $)$ & $L(\mathrm{Av})$ & $M_{*} / L(\mathrm{no} \mathrm{Av})$ & $M_{*} / L(\mathrm{Av})$ \\
\hline$B$ & $1.1 \times 10^{11}$ & $1.6 \times 10^{11}$ & 11.2 & 8.1 \\
555 & $1.5 \times 10^{11}$ & $2.2 \times 10^{11}$ & 7.8 & 5.9 \\
606 & $1.7 \times 10^{11}$ & $2.5 \times 10^{11}$ & 6.8 & 5.2 \\
775 & $1.5 \times 10^{11}$ & $2.2 \times 10^{11}$ & 7.7 & 6.0 \\
$H$ & $7.2 \times 10^{11}$ & $9.5 \times 10^{11}$ & 1.6 & 1.4 \\
\hline
\end{tabular}

The $B$ filter corresponds to the standard Bessell $B$ filter, and values are given in this filter in order to allow easier comparisons with other galaxies studied by different authors. All values are given in solar units. We report values corresponding to two different fits, with and without reddening respectively.

motivated by the goodness of the fit in this aperture $\left(\chi_{\text {d.o.f. }}^{2}<1\right)$. Moreover, we find the stellar mass to light ratios $\left(M_{*} / L\right)$ to be constant within this aperture. We use 8 evolutionary synthetic SEDs computed with the last version of the Bruzual \& Charlot (2003) code, with Chabrier (2003) IMF and solar metallicity. Given the data available, the photometric SED is equally well fit with either a very old elliptical template (a Single Stellar Population, SSP, aged 10.2 Gyr), or a slightly younger population (SSP aged 6-7 Gyr) including some reddening $\left(A_{\mathrm{V}}=0.4\right.$, with Calzetti et al. (2000) extinction law). The degeneracy in the $\left(A_{\mathrm{V}}\right.$, age $)$ plane yields slightly different results in the best fit template depending on the aperture radius. However, both types of models (i.e. with and without reddening) are equally likely, as shown in Fig. 4 for the adopted 7 " aperture. Although the total luminosity and $M_{*} / L$ ratio depend on the reddening correction, the stellar mass within a given aperture is found to be insensitive to the best fit template. The resulting stellar mass within a $7^{\prime \prime}$ aperture is $M_{*}=1.25 \pm 0.3 \times 10^{12} M_{\odot}$. According to Bell et al. (2007), the errors coming from setting the overall mass scale and its evolution are usually $25 \%$, much larger than the error on the photometry. We adopt $25 \%$ accuracy on the stellar mass estimation. Given the luminosity of this galaxy, we derive a a stellar mass to light ratio in the rest frame $B$ band equal to $\sim 8-11$ (solar units), depending on the reddening. We report all these values in Table 3. All luminosities are given in solar units calculated in the rest frame filters.

This is comparable (though a bit higher, but note that this galaxy has a very massive stellar population) to typical values of stellar mass to light ratio for giant elliptical galaxies (Gerhard et al. 2001).

\section{Multiply imaged systems}

Figure 1 shows a colour image of Abell 1703 where we label the multiply imaged systems. We report their positions and photometric redshifts in Table 1. The identification is a difficult step which is done in an iterative fashion: we begin to build a model using the most obvious lensed features. Then this model is used to test and predict possible multiply imaged systems. In total, we use 13 multiply imaged systems in this analysis. It is certain that more systems have to be found within the ACS field that presents many likely blue lensed features.

Here we give some notes on the different systems, and present colour images for each system in Appendix.

- System 1, the central ring: it is composed by 5 images. The four main bright images located close to the $\mathrm{cD}$ galaxy display a rare "hyperbolic umbilic" lensing configuration (see 


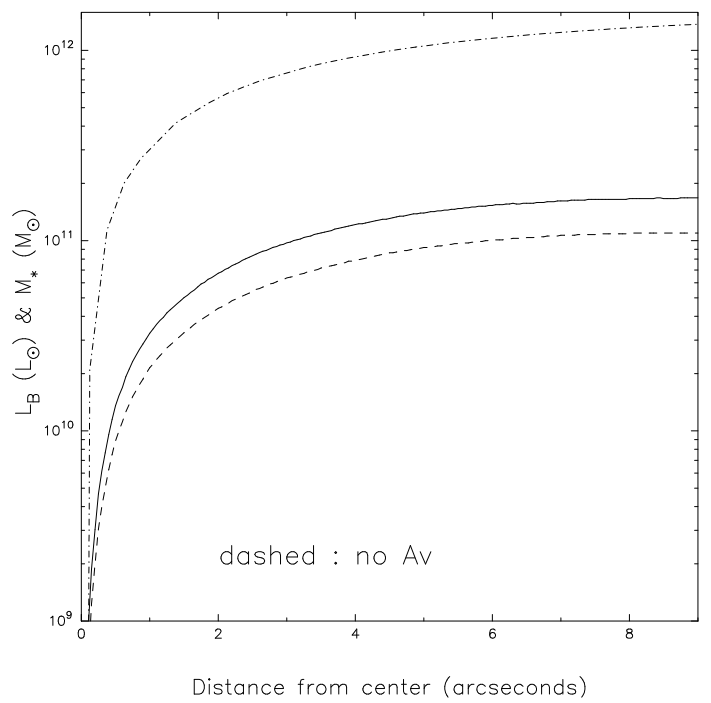

Fig. 3. Integrated luminosity profile of the $\mathrm{cD}$ galaxy, in the $B$ band rest frame, for the fit with reddening (solid) and without reddening (dashed), The corresponding mass profile used in the strong lensing analysis is shown as dot-dashed line. Both profiles have the same behaviour for $R>\sim 2^{\prime \prime}$.

Fig. 1 and Appendix). A demagnified counter image is located in the east. Image 1.3 is located at $5.5^{\prime \prime}=23 \mathrm{kpc}$ from the centre of the cluster, whereas image 1.1 is located at $13.5^{\prime \prime}=56 \mathrm{kpc}$ from the cluster centre. This system constitutes the innermost observational lensing constraint available in this analysis. This is the only system for which we have a spectroscopic redshift, equal to 0.8885 .

- System 2: this system is constituted by two images forming a straight gravitational arc. Two counter images are predicted to be more than two magnitudes fainter, we have not been able to detect any.

- System 3: system 3 is located in the northern part of the ACS field, close to Galaxy 852. It is composed of two merging bright images, and an additional fainter counter image a bit further west.

- System 4-5: we propose that a single lensed galaxy can be resolved into two parts, each part being considered as a multiply imaged system. It constitutes a typical cusp configuration system, with the images forming only on one side of the cluster.

- System 6: this is another cusp configuration system located a bit closer to the centre than system 4-5.

- Systems 7-8-9: these three cusp configuration systems are located in the north, at a radius a bit further from the centre than system 4-5.

- System 10-11: these two systems constitute the giant tangential arc. They correspond to two different spots we have identified on the giant arc that shows many substructures.

- System 15 and 16: these two systems composed by 4 images "follow" each other (Fig. 1): $15 . i$ and 16.i $(i=1,2,3,4)$ are found close to each other, presenting an Einstein cross configuration.

- Radial Arc: we report a radial feature coming out from the cD galaxy, composed by two spots (Fig. A.1). Due to the presence of the $\mathrm{CD}$, the estimated photometric redshift $(z \sim$ $1.4)$ is uncertain, and we have not been able to detect possible counterimages. This likely radial arc is not used in the analysis however.

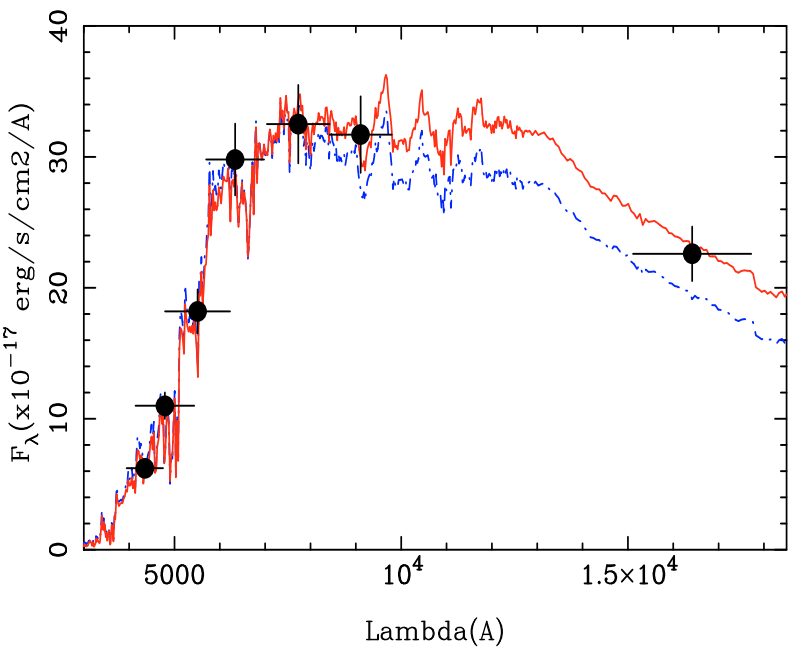

Fig. 4. Results of the SED fitting procedure. Luminosities are computed in an aperture of 7". The red solid line corresponds to the fit with reddening, and the blue dotted-dashed line corresponds to the fit without reddening. In each case, the fit is good $\left(\chi_{\text {d.o.f. }}^{2}<1\right)$ and leads to the same estimation of the stellar mass.

\section{Strong lensing analysis}

\subsection{Methodology}

To reconstruct the mass distribution in Abell 1703, we use a parametric method as implemented in the publicly available LENSTOOL $^{4}$ software (Jullo et al. 2007). We use the observational constraints (positions of the multiply imaged systems) to optimize the parameters used to describe the mass distribution: this is what we refer to as optimization procedure. The strong lensing methodology used in this analysis has been described in details in Limousin et al. (2007b). We refer the interested reader to this article for a complete description of our methodology.

We describe the mass distribution in Abell 1703 by constructing a two components mass model: the contribution from the dominant central $\mathrm{cD}$ galaxy is fixed by its stellar mass, and then the remaining mass is put into an underlying smooth dark matter distribution described using a generalized NFW profile (see Sand et al. 2008, for details on the implementation of the generalized NFW profile into the LENSTOOL code). We also take into account the perturbations associated with the galaxies.

\subsection{Modelling the $c D$ contribution}

Degeneracies can arise between the two mass components: if too much mass is put into the $\mathrm{cD}$ galaxy, then this can lead to a shallower slope of the dark matter halo and vice versa. Therefore, special care has to be taken when modelling the $\mathrm{cD}$ galaxy, and such a modelling must be as much as possible "observationally motivated". We use a dual Pseudo Isothermal Elliptical Mass Distribution (dPIE, see Elíasdóttir et al. 2007) with no core radius to describe the $\mathrm{cD}$ stellar mass contribution. This profile is formally the same as the Pseudo Isothermal Elliptical Mass Distribution (PIEMD) profile described in Limousin et al. (2005). However, as explained in Elíasdóttir et al. (2007) it is not the same as the PIEMD originally defined by Kassiola \& Kovner (1993). Therefore we have adopted the new name dPIE to avoid confusion. The position of this mass clump is fixed at $(0,0)$; the ellipticity and position angle are set to be the one we measured

\footnotetext{
${ }^{4}$ http://www.oamp.fr/cosmology/lenstool/
} 
from the light distribution. Given this parametrization, the mass of the $\mathrm{cD}$ scales as $M_{\mathrm{cD}} \propto \sigma_{0}^{2} \times r_{\text {cut }}$, where the scale radius $r_{\text {cut }}$ is almost equal to the half mass radius, and $\sigma_{0}^{2}$ is a fiducial velocity dispersion (Elíasdóttir et al. 2007). The choice of the scale radius will influence the shape of the mass profile. The smaller the scale radius, the steeper the mass profile. We choose a scale radius of $25 \mathrm{kpc}$ so that the mass profile is as close as possible to the integrated luminosity profile. As we can see in Fig. 3, the mass profile and the luminosity profile have the same behaviour for $R>\sim 2^{\prime \prime}$. Note that $25 \mathrm{kpc}$ is close to the value used by Sand et al. (2008) to describe the cD galaxies in MS 2137 (22 kpc) and Abell 383 (26 kpc), where the same mass profile has been used. The only free parameter describing this $\mathrm{cD}$ galaxy is then $\sigma_{0}$. This parameter is allowed to vary in a range that is set by the choice of the scale radius and the estimation of the stellar mass.

\subsection{Modelling the underlying dark matter distribution}

We assume that the dark matter component can be described using a generalized NFW model. Its $3 \mathrm{D}$ mass density profile is given by:

$\rho(r)=\frac{\rho_{\mathrm{c}} \delta_{\mathrm{c}}}{\left(r / r_{\mathrm{s}}\right)^{\alpha}\left(1+\left(r / r_{\mathrm{s}}\right)\right)^{3-\alpha}}$,

with $r_{\mathrm{s}}$ the scale radius (note that the scale radius for an NFW profile does not have the same meaning as the scale radius for a dPIE profile, see Limousin et al. 2005). For a galaxy cluster, this parameter is supposed to be larger than $150 \mathrm{kpc}$ (Sect. 4.5), so in the range in radius we probe in this work, we have $r<r_{\mathrm{s}}$ and thus the density profile can be approximated by $\rho(r) \sim r^{-\alpha}$.

Note that we have subtracted only the stellar contribution of the $\mathrm{cD}$ galaxy in our modelling. This is consistent with the general picture that the dark matter halo of the cluster is also the one of the cD galaxy (see, e.g. Miralda-Escude 1995). We cannot distinguish both haloes.

\subsection{Galaxy scale perturbers}

On top of these two components, we include the brightest cluster members in the optimization (Sect. 2.3). This galaxy scale component is incorporated into the modelling using empirical scaling relations that relate their dynamical parameters (central velocity dispersion and scale radius) to their luminosity, whereas their geometrical parameters (centre, ellipticity, position angle) are set to the one measured from the light distribution (see Limousin et al. 2007b, for details). This galaxy scale component is thus parametrized by only two free parameters, and at the end of the optimization procedure, we get constraints on the parameters for a galaxy of a given (arbitrary) luminosity which corresponds to an observed magnitude $m_{F 775 W}=18.3$.

One single galaxy (labeled 852 in our catalogue) is optimized individually, in the sense that some of the parameters describing this galaxy are allowed to vary instead of being fixed by its luminosity. This was necessary to reproduce better the geometrical configuration of some images falling close to this galaxy (systems 3, 7, 8 and 9). In fact, a visual inspection at the image of the cluster shows that this galaxy is very bright and extended (Fig. 1). This galaxy is not representative of the cluster galaxy population, thus the adopted scaling laws might not apply to this object. Moreover, as shown in Appendix, a likely lensed blue feature is coming out from this galaxy, suggesting a massive substructure.

\subsection{Limits on the parameters}

Each parameter is allowed to vary between some limits (priors). The position of the DM clump was allowed to vary between $\pm 25^{\prime \prime}$ along the $X$ and $Y$ directions. Its ellipticity was forced to be lower than 0.5 , since beyond that, the ellipticity as defined in this work for an NFW profile is no longer valid (Golse \& Kneib 2002). Its slope $\alpha$ was allowed to vary between 0.2 and 2.0 and its scale radius $r_{\mathrm{s}}$ between 150 and $750 \mathrm{kpc}$ (Tasitsiomi et al. 2004; Dolag et al. 2004). The concentration parameter $c_{200}$ was allowed to vary between 2.5 and 9, which is large enough to include the expectations from the most recent results from $N$-body simulations (Neto et al. 2007). The mass of the cD component was forced to be within the range allowed by the stellar mass estimate. Concerning the galaxy scale component, we allowed the velocity dispersion to vary between 150 and $250 \mathrm{~km} \mathrm{~s}^{-1}$, and the scale radius was forced to be smaller than $70 \mathrm{kpc}$, since we have evidence both from observations (Natarajan et al. 1998; Geiger \& Schneider 1999; Natarajan et al. 2002a,b; Limousin et al. 2007a; Halkola et al. 2007) and from numerical simulations (Limousin et al. 2007c) that dark matter haloes of cluster galaxies are compact due to tidal stripping.

\section{Results}

\subsection{Mass distribution from strong lensing}

Results of the optimization ${ }^{5}$ are given in Table 4. The images are well reproduced by our mass model, with an image plane rms equal to $1.4^{\prime \prime}\left(0.2^{\prime \prime}\right.$ in the source plane). rms for individual systems are listed in Table 1 . We derive from our model a 2D projected mass within $50^{\prime \prime}$ equal to $M\left(50^{\prime \prime}\right)=2.4 \times 10^{14} M_{\odot}$.

Dark matter component: the logarithmic slope of the 3D dark matter distribution is found to be equal to $1.09_{-0.11}^{+0.05}$ ( $3 \sigma$ confidence level). We show in Fig. 7 degeneracy plots between $\alpha$ and: the mass of the $\mathrm{cD}$ galaxy, the concentration parameter $c_{200}$, and the scale radius $r_{\mathrm{s}}$. We find $c_{200} \sim$ [3.0-4.2] and $M_{200} \sim 1.8 \times 10^{15} M_{\odot}$. However, we caution that these values rely on pure extrapolation from the strong lensing fit and that these quantities should be probed using weak lensing and/or $\mathrm{X}$-ray data. Indeed, the NFW scale radius is found to be larger than the radius over which we have observational constraints (i.e. $\sim 54^{\prime \prime}$ ). The ellipticity of the mass distribution is low, with $a / b=1.13$. The location of the DM clump coincide with the position of the $\mathrm{cD}$ galaxy. No second large scale dark matter clump was needed by the data. From previous experience modelling bimodal galaxy clusters such as Abell 1689, Abell 2218, Abell 68 and MS 2053.7-0449, we are pretty confident that there is no need for a second large scale DM clump.

Galaxy scale perturbers: the parameters inferred for the galaxy population (for an observed magnitude equal to 18.3 in the $F 775$ band) are: $\sigma_{0}=210 \pm 4 \mathrm{~km} \mathrm{~s}^{-1}$, and $r_{\text {cut }}=66 \pm 5 \mathrm{kpc}$. We checked that the degeneracies between these two parameters are the expected ones, in the sense that they follow constant mass

\footnotetext{
5 A parameter file containing all the following information, and which can be used with the publicly available LENSTOOL software, is available at http://www.dark-cosmology.dk/archive/. This file can be useful for making model based predictions, e.g. counter-images of a multiple image candidate, amplification and mass map and location of the critical lines at a given redshift, and it will be updated.
} 
Table 4. Mass model parameters.

\begin{tabular}{ccccccccc}
\hline \hline Clump & $\delta(x)$ & $\delta(y)$ & $e$ & $\theta$ & $r(\mathrm{kpc})$ & $\alpha$ & $c_{200}$ & $\sigma_{0}\left(\mathrm{~km} \mathrm{~s}^{-1}\right)$ \\
\hline NFW & $-1.0 \pm 0.1$ & $0.5 \pm 0.1$ & $0.12 \pm 0.004$ & $64.5 \pm 0.3$ & $727.0_{-76.77}^{+13.84}$ & $1.09_{-0.036}^{+0.015}$ & $3.1_{-0.07}^{+0.34}$ & - \\
CD & {$[0.0]$} & {$[0.0]$} & {$[0.22]$} & {$[52]$} & {$[30]$} & - & - & $299.4_{-9.9}^{+0.35}$ \\
Galax 852 & {$[19.0]$} & {$[54.0]$} & {$[0.11]$} & {$[65.5]$} & $97.1 \pm 1.2$ & - & - & $319.5 \pm 4.1$ \\
$L^{*}$ elliptical galaxy & - & - & - & - & $65.9_{-4.6}^{+0.16}$ & - & - & $207.8_{-15.0}^{+18.0}$ \\
\hline
\end{tabular}

Coordinates are given in arcseconds with respect to the cD Galaxy. The ellipticity $e$ is the one of the mass distribution, expressed as $a^{2}-b^{2} / a^{2}+b^{2}$. Error bars correspond to $1 \sigma$ confidence level as inferred from the MCMC optimization. When the posterior probability distribution is not Gaussian, we report the mode and asymmetric error bars. Values into brackets are not optimized. Note that the meaning of the scale radius reported here is different for the generalized NFW clump and the other mass clumps, described using a dPIE profile with no core.

lines $\left(M \propto \sigma_{0} \times r_{\text {cut }}\right)$. Galaxy 852 is found to be quite massive, with $M\left(15^{\prime \prime}\right) \sim 5 \times 10^{12} M_{\odot}$.

\subsection{Mass and light: a relaxed unimodal cluster?}

We compare the mass and the light distribution in Fig. 6. We find they do compare well, suggesting that light traces mass in Abell 1703. Also shown is the light distribution from the dominant cD galaxy, which is found to be consistent with the one of the overall mass distribution within a few degrees. Moreover, we find the centre of the DM halo to be coincident with the centre of the cD galaxy.

At first approximation, these facts suggest that Abell 1703 is a relaxed unimodal cluster. This hypothesis should be investigated further, in particular with precise X-ray observations since we expect the X-ray emission to be centred on the $\mathrm{cD}$ galaxy, and to present a low ellipticity.

Besides, we can draw a line from the southern part of the ACS field up to its northern part that pass through the $\mathrm{cD}$ galaxy and that connects very bright galaxies, much brighter than the overall galaxy population (Fig. 1). Interestingly, when looking at Abell 1703 on much larger scales from SDSS imaging (which size is $581^{\prime \prime} \times 809^{\prime \prime}$ ), the whole cluster galaxy population looks rather homogeneous, with no very luminous galaxies as we can observe along this filamentary structure. In the south of the ACS field, this structure seem to have an influence on the formation of the giant arc, breaking its symmetry (Appendix). The perturbation associated with the northern part of this structure is more obvious to detect. Indeed, the formation of systems 3, 7, 8, and 9 (both their existence and their geometrical configuration) is connected with this extra mass component. Moreover, we can see that a blue lensed feature is coming out from Galaxy 852 (Appendix). The fact that we had to constrain individually Galaxy 852 also points out that some extra mass is needed in this region and that this galaxy (and possibly the other bright galaxies defined by this filament) is not representative of the overall cluster population. One tentative explanation could be that we are observing a galaxy group infalling in the cluster centre. Though this scenario would need a devoted spectroscopic follow up of the cluster members to get some insights into the velocity dimension of Abell 1703.

\subsection{Reliability of the constraints on $\alpha$}

One of the main goals of this work is to measure the slope of the underlying dark matter component, parametrized by $\alpha$. We want to stress out again that we have assumed that the underlying mass distribution can be described using a generalized NFW profile, but this assumption may not be correct. We try some tests in order to check the reliability of our measurement.
CD modelling: as discussed before, $\alpha$ will be sensitive to the parametrization of the $\mathrm{cD}$ galaxy. During the first steps of this work, we noticed that if too much mass is put into the cD component, then $\alpha$ can become smaller than what is found here, and vice versa: if not enough mass is put into the $\mathrm{cD}$ component, $\alpha$ can become larger. However, these scenarios were leading to stellar masses that are not compatible with the photometry. Remain the choice of the scale radius of the $\mathrm{cD}$ galaxy. The value used in this analysis is set by the shape of the integrated luminosity profile. We tried to use lower (down to $10 \mathrm{kpc}$ ) and higher (up to $45 \mathrm{kpc}$ ) values of the scale radius, tuning up or down the velocity dispersion to keep the stellar mass of the $\mathrm{cD}$ consistent with what we have estimated from the broad band photometry. We find this does not have strong influence on the estimation of $\alpha$. More precisely, when using a scale radius of $10 \mathrm{kpc}$, we find $\alpha=1.10 \pm 0.04$, whereas when using a scale radius of $45 \mathrm{kpc}$, we find $\alpha=1.05 \pm 0.05$.

System 1: system 1 constitutes the innermost observational constraint in our analysis. The configuration of the brightest 4 images forming the central ring allows us to probe the potential at small radii and this system constitutes our most stringent constrain: if removing system 1 from the analysis, we found that the slope was basically unconstrained, since we could get an equal fit with $\alpha \sim 0.5$ or $\alpha \sim 1.5$, suggesting that the use of a generalized NFW profile was not necessary. We note that the rms of system 1 is slightly larger than the mean total rms. This interesting lensing feature is not perfectly retrieved in our analysis. One could try to improve the situation by considering the influence of galactic substructures on the formation of this ring. However, from the large separation of the counter image 1.5, it is clear that this central ring is not a galaxy-scale lens. System 1 is also the only system for which we have been able to measure a spectroscopic redshift. This allows us to check how the results presented in this work are dependent of the redshift of this system. Instead of fixing its redshift to the measured one, $z=0.8885$, we allowed it to vary between 0.72 and 1.0 as constrained from the photometry. In this case, the model slightly underestimate the redshift and find $z \sim 0.78$. The slope of the underlying dark matter halo shifts to higher values, $\alpha \sim 1.3$. This highlight the importance of having spectroscopic redshifts over photometric redshifts when it comes to detailed study of mass distributions.

Photometric redshifts: as we can appreciate in Table 1, most of the photometric redshifts are well constrained by the available filters. In particular, the redshift probability functions were dominated by a well defined peak. In order to investigate the importance of using reliable photometric redshifts, we redid the analysis as follows: the redshift of system 1 was fixed to the 
spectroscopically measured value, and all other redshifts where assigned a flat prior between 0.28 (redshift of the cluster) and 6 . Results of the optimized redshifts $\left(z_{\text {free }}\right)$ are given in Table 1. We can see that they differ significantly from their photometrically constrained values, and that they are systematically overestimated. Results on the parameters of the dark matter clump are the following: $\alpha=1.22_{-0.05}^{+0.03} ; c_{200}=3.4 \pm 0.3 ; r_{\mathrm{s}} \sim 520 \mathrm{kpc}$. Therefore, mainly the value of the slope is significantly changed when letting redshifts being free. Since increasing $\alpha$ will lead to decreasing the mass enclosed in a given radius, redshifts are being overestimated in order to compensate for. This highlight the importance of photometric redshift when spectroscopic redshift are not available.

X-ray gas component: in this analysis, we have not been able to subtract the mass contribution from the X-ray gas. Recent analysis by Bradač et al. (2007) in RXJ 1347.5-1145, combining lensing and X-ray, has been able to disentangle each component. Indeed, they found that the total mass profile (DM+gas+stars) is slightly higher than the DM mass profile, the ratio of both quantities at $75 \mathrm{kpc}$ being equal to $85 \%$. This could suggest that one can neglect the gas component at first approximation. Of course, Abell 1703 and RXJ 1347.5-1145 look very different thus we cannot compare them quantitatively. To be more precise, we would need devoted X-ray observations of Abell 1703.

Limits on the parameters: the limits adopted in this work are motivated by results from numerical simulations. Here we want to investigate further what is the influence of the adopted limits on the results, in particular on the slope of the dark matter distribution. First, we released these limits for $c_{200}$. When allowed to vary between 1 and 13 (instead of 2.5 and 9), we find the parameters of the dark matter distribution to be fully consistent with the results given in Table 4 .

Another concern is the scale radius $r_{\mathrm{s}}$. We find it to be larger than the range within which observational constraints are available. Since degeneracies arise between the scale radius and the slope of the generalized NFW profile, we redid the analysis by imposing the scale radius to be within different limits in order to see the influence on the results. Since we do have observational constraints up to $210 \mathrm{kpc}$ from the centre of the cluster, we are confident that if $r_{\mathrm{s}}$ was below $200 \mathrm{kpc}$, our analysis would have been able to constrain it. Therefore, we consider the following ranges: (1) 200-300 kpc; (2) 300-400 kpc; (3) 400-600 kpc and (4) 600-800 kpc. We report the results in Table 5: (0) corresponds to the mass model presented in Sect. 5.1 and Table 4. The following lines correspond to models obtained when assuming a different range of limits for the scale radius. For each model $(i)$, we report the inferred values for $\left(r_{\mathrm{s}}, c_{200}, \alpha\right)$ and compare each run with model $(0)$. In order to quantify this comparison, we report $\Delta\left(\log \left(E_{\mathrm{v}}\right)\right)$ which is the difference between the Bayesian Evidence of model (0) and the Bayesian Evidence of the considered model. When this quantity is positive, it favours model (0) as being more likely. We also report $\Delta\left(\chi^{2}\right)$ which is the difference between the $\chi^{2}$ of model (0) and the $\chi^{2}$ of the considered model. When this quantity is negative, it favours model (0) as being a better fit to the data.

We can draw the following conclusions: for models (1), (2) and (3), we see that the scale radius is always found at the higher end of the allowed limit, suggesting that this parameter could be larger than the upper limit assigned. As a result, we find these models to be less likely than the model (0), since both their $\chi^{2}$ and Bayesian Evidence are worse. The results for model (4) are
Table 5. Results of the analysis when using different limits for the scale radius $r_{\mathrm{s}}$.

\begin{tabular}{cccccc}
\hline \hline Limits (kpc) & $r_{\mathrm{s}}(\mathrm{kpc})$ & $c_{200}$ & $\alpha$ & $\Delta\left(\log \left(E_{\mathrm{v}}\right)\right)$ & $\Delta\left(\chi^{2}\right)$ \\
\hline (0) $150-750$ & $727.0_{-76.77}^{+13.84}$ & $3.1_{-0.07}^{+0.34}$ & $1.09_{-0.036}^{+0.015}$ & - & - \\
(1) $200-300$ & $299.5_{-3.28}^{+0.09}$ & $6.9_{-0.12}^{+0.16}$ & $0.77_{-0.021}^{+0.032}$ & 19.6 & -41 \\
(2) $300-400$ & $398.8_{-3.28}^{+0.09}$ & $5.4_{-0.12}^{+0.20}$ & $0.91_{-0.032}^{+0.020}$ & 8.9 & -19 \\
(3) $400-600$ & $598.8_{-41.27}^{+1.27}$ & $3.7_{-0.08}^{+0.24}$ & $1.03_{-0.021}^{+0.024}$ & 1.9 & -4 \\
(4) 600-800 & $719.3_{-61.96}^{+22.94}$ & $3.0_{-0.01}^{+0.32}$ & $1.09_{-0.026}^{+0.019}$ & -3.9 & -1 \\
\hline
\end{tabular}

Error bars correspond to $1 \sigma$ confidence level as inferred from the MCMC optimization. When the posterior probability distribution is not Gaussian, we report the mode and asymmetric error bars. (0) corresponds to the results of the mass model presented in Table 4. The following lines corresponds to models obtained when assuming a different prior for the scale radius. For each model $(i)$, we report the results on the parameters of the dark matter clump. To compare the different lines, we report $\Delta\left(\log \left(E_{\mathrm{v}}\right)\right)$ and $\Delta\left(\chi^{2}\right)$.

essentially the same as for model (0): the parameters, as well as the $\chi^{2}$ are found to be fully consistent. The Bayesian Evidence favours model (4) over model (0), which can be understood by the fact that the ratio between the posterior and the prior is smaller in the case of model (4). Note that model (3) is also consistent with model $(0)$, in the sense that the mass clump parameters agree with each other, and that both Evidences and $\chi^{2}$ are comparable.

These results suggest that, even though the preferred value for $r_{\mathrm{s}}$ is found larger than the range over which observational constraints are found, the multiple images actually are sensitive to the value of $r_{\mathrm{s}}$. This can be understood as follows: the generalized NFW profile is not a power law model, in the sense that the 3D density $\rho(r)$ is changing at each radius $r$. This mass profile will be close to isothermal for $r \sim r_{\mathrm{s}}$, and the radius at which the profile becomes isothermal could be felt by observational constraints located at $r<r_{\mathrm{s}}$. To check this scenario, we compute the 2D aperture masses inferred from each model and compare them. If there is a significant mass difference between each model at the radius where observational constraints are present, then we could understand why the observational constraints are able to discriminate between each model. Comparison between masses, expressed as a percentage (calculated as $(\operatorname{model}(0)-\operatorname{model}(1)) / \operatorname{model}(0))$, is shown in Fig. 5 . We see that mass differences can reach up to $3 \%$ at the radius where the outermost observational constraint is found $(\sim 210 \mathrm{kpc})$. The question is to know whether we are sensitive to such a mass difference. In other words: is the accuracy on our mass measurement below this mass differences? From the MCMC realizations, we estimate the accuracy on our mass measurement and express it as a percentage. This accuracy depends on the distance from the cluster centre, and is plotted in Fig. 5. If mass differences are below the accuracy on our mass measurement up to $\sim 125 \mathrm{kpc}$, we see that for $R>150 \mathrm{kpc}$, mass differences between model (0) and models (1) and (2) are above the accuracy on the mass measurement, which means that we are able to discriminate between these different mass models. This shows that the multiple images located further away than $150 \mathrm{kpc}$ from the centre are sensitive to the value of $r_{\mathrm{s}}$.

Ultimately, the scale radius of Abell 1703 should be constrained by a careful strong and weak lensing and/or X-ray analysis. 


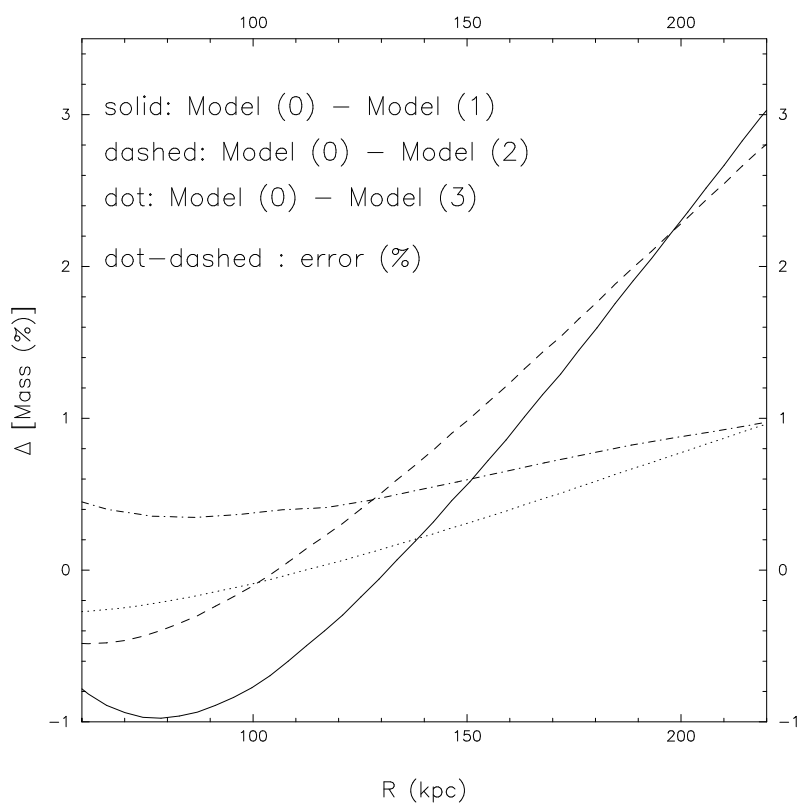

Fig. 5. Mass differences between model (0) and model (1) (solid), model (2) (dashed) and model (3) (dotted). The dot-dashed line correspond to the accuracy on the mass measurement, expressed as a percentage. If mass differences are below the accuracy up to $\sim 125 \mathrm{kpc}$, we see that for $R>150 \mathrm{kpc}$, mass differences between model (0) and models (1) and (2) are above the accuracy on the mass measurement, which means that we are able to discriminate between these different mass models.

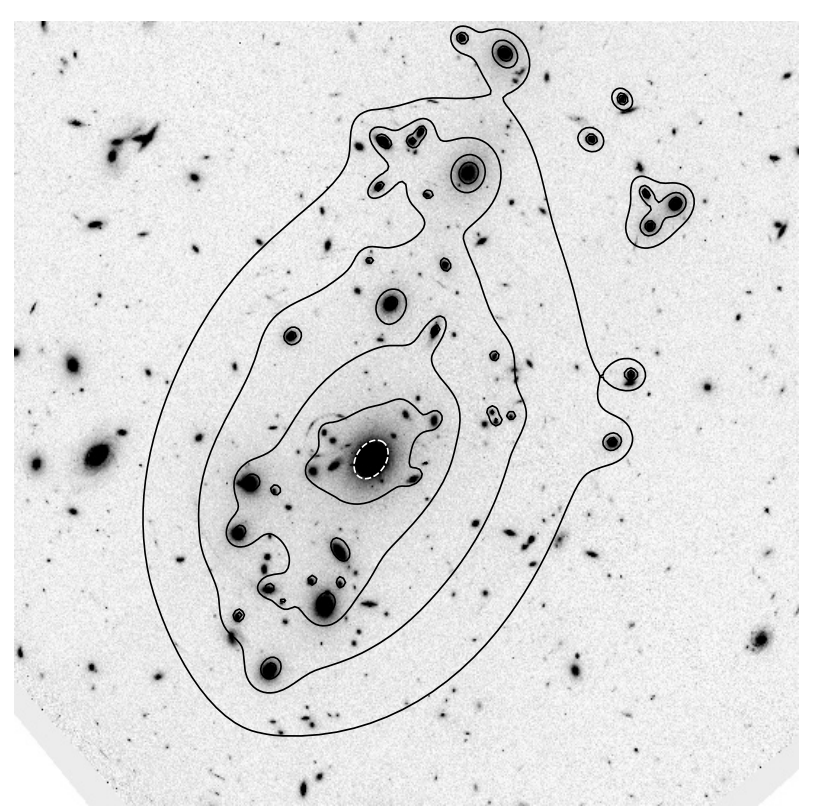

Fig. 6. Mass contours overlaid on the ACS F850W frame. Also shown is the light distribution from the $\mathrm{cD}$ galaxy (dashed white contours), which is found to be consistent in orientation with the one of the mass distribution within a few degrees. Note how the light and the mass follow the filamentary structure. Size of panel is $153^{\prime \prime} \times 153^{\prime \prime}$.

\section{Discussion}

The main result of the presented work is to measure a central slope equal to $\sim-1.1$. This is close to the predictions from Navarro et al. (1997). However, we want to stress again that comparing these values is not relevant since dark matter only simulations, by definition, do not take into account the likely influence from the baryonic component on the shape of the underlying dark matter, thus their predictions cannot be reliably compared to what is inferred observationally.

Similar analyses (i.e. lensing analyses aiming to probe the central mass density distribution in galaxy clusters) have been carried out by Sand et al. (2002, 2004, 2008) (see also Gavazzi et al. 2003; Gavazzi 2005, on MS 2137-23). Studies by Sand et al. used the measured velocity dispersion profile of the cD galaxy as an extra constrain. The first work by Sand et al. (2004) assumed a circular cluster. As shown by Meneghetti et al. (2007b), this assumption was likely to bias the results towards shallower values of the central mass density slope. Then Sand et al. (2008) redid their analysis using a full 2D lensing analysis, taking into account the presence of substructures and allowing the clusters for non circularity. They confirmed their earlier claims, in particular, they do find evidence for a central mass density slope to be less than 1 . The results presented in this work points out towards higher values for the slope of the central mass distribution compared to the studies by Sand et al. It is worth mentioning that our analysis is close to the ones by Sand et al., since we have both used strong lensing techniques, and moreover used almost the same LENSTOOL software (by the time of the studies by Sand et al., the Bayesian Monte Carlo Markov Chain sampler as described by Jullo et al. 2007, was not available, and they used a parabolic $\chi^{2}$ optimization instead, but this cannot explain the differences). This wide range of slopes found from one cluster to another may point out to an intrinsic large scatter on this parameter, which may depend on the merger history of the cluster. This possible scatter should be probed numerically.

Prospects on Abell 1703: we can summarize some possible extensions of the presented work:

1. We have left some blue features that are likely to be lensed. Therefore, the multiply imaged systems identification is still to be improved. In particular, it would be useful to understand the radial feature that is coming out from the cD galaxy.

2. Parametric strong lensing is very sensitive to misidentifications, thus we are pretty confident of our identifications. However, a measure of their redshifts would be very valuable.

3. Probing the cluster potential on larger scales: our analysis allowed the scale radius to vary within a large prior suggested by numerical simulations. We have investigated how the choice of this prior can influence the results on $\alpha$, finding substantial differences between different priors. Measuring reliably the scale radius is thus of first importance and should be done combining strong and weak lensing and/or X-ray measurements.

4. Measuring the velocity dispersion of the stars of the $\mathrm{cD}$ galaxy in order to add dynamical constraints as in the studies by Sand et al.

5. The more relaxed Abell 1703, the stronger the conclusions of the work presented here, and also the easier the comparison with $N$-body simulations. We have argued above that we find evidence for a relaxed cluster. It would be important to check further the dynamical state of Abell 1703. In particular, high resolution X-ray observations of Abell 1703 could help to answer this question. Moreover, X-ray observations would also make possible to subtract the gas contribution from the mass budget, following Bradač et al. (2007).

6. Moreover, the spectroscopic study of the motions of cluster galaxies provides clues to the dynamical state of the 

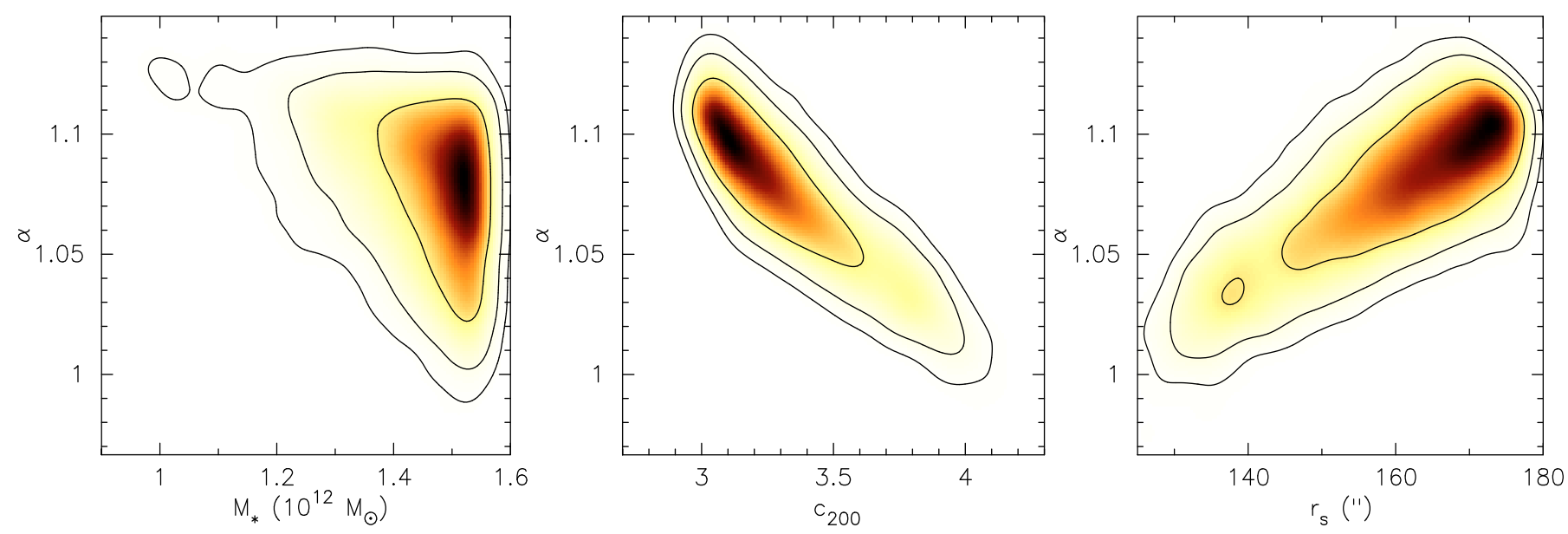

Fig. 7. Degeneracy plots between $\alpha$ and (from left to right): cD stellar mass, $c_{200}$ and $r_{\mathrm{s}} . \alpha$ corresponds to the logarithmic slope of the underlying dark matter mass distribution parametrized by a generalized NFW profile. $c_{200}$ and $r_{\mathrm{s}}$ correspond to the concentration parameter and the scale radius respectively for this mass distribution. Note that the scale radius is found larger than the range within which we have observational constraints.

cluster. Such data give an insight into the velocity dimension, and can reveal if the cluster is undergoing a merger along the line of sight or if it is already well relaxed. Of particular interest will be to probe the velocity difference between the $\mathrm{cD}$ galaxy and the brightest cluster galaxies defining this filamentary structure, in order to investigate if we are observing an infalling galaxy group.

7. Related to this group issue is the study of Galaxy 852 based on the detection of a nearby arc and the surrounding multiply imaged systems.

Prospects on the Central Mass Distribution of Galaxy Clusters: obviously, we suffer from small number statistics and we need to extend this kind of analyses to other clusters. In trying to gather a sample of clusters for which the presented analysis could be applied, one should focus on relaxed unimodal $\mathrm{cD}$ dominated clusters which present observational constraints as close as possible to the centre of the cluster. In that respect, one should look for radial arcs. Note, however, that radial arcs might preferentially form in clusters with shallow profile. Ideally, selection should be independent of arc appearances, but the clusters with radial arcs will indeed give good constraints.

On the numerical side, we need to study a sample of many galaxy clusters containing baryons, and testing different prescriptions for the baryonic implementation. We have initiated such a numerical study on two galaxy clusters, and results will be presented in a forthcoming publication.

Conducting in parallel an observational and a numerical program is a worthy goal: it is interesting by itself to study what is going on in the central part of the most massive virialized structures since it can provide insights on the interactions between baryons and dark matter particles; moreover, it can potentially provide an interesting probe of cosmological models.

Acknowledgements. We thank many people for constructive comments and discussion related to this topic, in particular: Bernard Fort, Dave Sand, Hans Böhringer, Jens Hjorth, Kristian Pedersen, Steen Hansen. We thank John Stott for creating the colour image from which Fig. 1 has been made, and for allowing us to use it. The referee is acknowledged for a careful reading and a constructive report. M.L. acknowledges the Agence Nationale de la Recherche for its support, project number BLAN06-3-135448. The Dark Cosmology Center is funded by the Danish National Research Foundation. J.R. is grateful to Caltech for its support. J.P.K. aknowledges the Centre National de la Recherche Scientifique for its support. We thank the Danish Centre for Scientific Computing at the University of Copenhagen for providing us generous amount of time on its supercomputing facility. Based on data collected at Subaru Telescope, which is operated by the National Astronomical Observatory of Japan. We are thankful to Ichi Tanaka for his support in the reduction of MOIRCS imaging data. M.L. acknowledges the lensing group at Shanghai Normal University for their kind invitation and hospitality, during which this work has been initiated. The authors recognize and acknowledge the very significant cultural role and reverence that the summit of Mauna Kea has always had within the indigenous Hawaiian community. We are most fortunate to have the opportunity to conduct observations from this mountain.

\section{References}

Allen, S. W., Edge, A. C., Fabian, A. C., et al. 1992, MNRAS, 259, 67 Arabadjis, J. S., Bautz, M. W., \& Garmire, G. P. 2002, ApJ, 572, 66 Austin, C. G., Williams, L. L. R., Barnes, E. I., Babul, A., \& Dalcanton, J. J. 2005, ApJ, 634, 756

Bell, E. F., Zheng, X. Z., Papovich, C., et al. 2007, ApJ, 663, 834

Bertin, E., \& Arnouts, S. 1996, A\&A, 117, 393

Biviano, A., \& Salucci, P. 2006, A\&A, 452, 75

Blumenthal, G. R., Faber, S. M., Flores, R., \& Primack, J. R. 1986, ApJ, 301, 27

Böhringer, H., Voges, W., Huchra, J. P., et al. 2000, ApJS, 129, 435

Bolzonella, M., Miralles, J.-M., \& Pelló, R. 2000, A\&A, 363, 476

Bradač, M., Schrabback, T., Erben, T., et al. 2007, [arXiv:0711.4850]

Bruzual, G., \& Charlot, S. 2003, MNRAS, 344, 1000

Calzetti, D., Armus, L., Bohlin, R. C., et al. 2000, ApJ, 533, 682

Casertano, S., de Mello, D., Dickinson, M., et al. 2000, AJ, 120, 2747

Chabrier, G. 2003, PASP, 115, 763

Coleman, D., Wu, C., \& Weedman, D. 1980, ApJS, 43, 393

Dahle, H., Hannestad, S., \& Sommer-Larsen, J. 2003, ApJ, 588, L73

Dolag, K., Bartelmann, M., Perrotta, F., et al. 2004, A\&A, 416, 853

El-Zant, A., Shlosman, I., \& Hoffman, Y. 2001, ApJ, 560, 636

El-Zant, A. A., Hoffman, Y., Primack, J., Combes, F., \& Shlosman, I. 2004, ApJ, 607, L75

Elíasdóttir, Á., Limousin, M., Richard, J., et al. 2007, [arXiv:0710. 5636]

Ettori, S., Fabian, A. C., Allen, S. W., \& Johnstone, R. M. 2002, MNRAS, 331, 635

Gao, L., Navarro, J. F., Cole, S., et al. 2008, MNRAS, 387, 536

Gavazzi, R. 2005, A\&A, 443, 793

Gavazzi, R., Fort, B., Mellier, Y., Pelló, R., \& Dantel-Fort, M. 2003, A\&A, 403, 11

Geiger, B., \& Schneider, P. 1999, MNRAS, 302, 118

Gerhard, O., Kronawitter, A., Saglia, R. P., \& Bender, R. 2001, AJ, 121, 1936

Ghigna, S., Moore, B., Governato, F., et al. 2000, ApJ, 544, 616

Gnedin, O. Y., Kravtsov, A. V., Klypin, A. A., \& Nagai, D. 2004, ApJ, 616, 16

Golse, G., \& Kneib, J.-P. 2002, A\&A, 390, 821

Gustafsson, M., Fairbairn, M., \& Sommer-Larsen, J. 2006, Phys. Rev. D, 74, 123522

Halkola, A., Seitz, S., \& Pannella, M. 2007, ApJ, 656, 739

Hansen, S. H., \& Stadel, J. 2006, J. Cosmol. Astro-Part. Phys., 5, 14

Ichikawa, T., Suzuki, R., Tokoku, C., et al. 2006, in Ground-based and Airborne Instrumentation for Astronomy, Presented at the Society of Photo-Optical 
Instrumentation Engineers (SPIE) Conf., ed. McLean, Ian S., Iye, Masanori. Proc. SPIE, 626916

Jullo, E., Kneib, J.-P., Limousin, M., et al. 2007, New J. Phys., 9, 447

Kassiola, A., \& Kovner, I. 1993, ApJ, 417, 450

Kazantzidis, S., Magorrian, J., \& Moore, B. 2004, ApJ, 601, 37

Kelson, D. D., Zabludoff, A. I., Williams, K. A., et al. 2002, ApJ, 576, 720

Kinney, A. L., Calzetti, D., Bohlin, R., et al. 1996, ApJ, 467, 38

Kneib, J., Hudelot, P., Ellis, R. S., et al. 2003, ApJ, 598, 804

Lewis, A. D., Buote, D. A., \& Stocke, J. T. 2003, ApJ, 586, 135

Limousin, M., Kneib, J.-P., \& Natarajan, P. 2005, MNRAS, 356, 309

Limousin, M., Kneib, J. P., Bardeau, S., et al. 2007a, A\&A, 461, 881

Limousin, M., Richard, J., Jullo, E., et al. 2007b, ApJ, 668, 643

Limousin, M., Sommer-Larsen, J., Natarajan, P., \& Milvang-Jensen, B. 2007c, [arXiv:0706.3149]

Ma, C.-P., \& Boylan-Kolchin, M. 2004, Phys. Rev. Lett., 93, 021301

Madau, P. 1995, ApJ, 441, 18

Mandelbaum, R., Seljak, U., \& Hirata, C. M. 2008, [arXiv: 0805 . 2552]

Meneghetti, M., Argazzi, R., Pace, F., et al. 2007a, A\&A, 461, 25

Meneghetti, M., Bartelmann, M., Jenkins, A., \& Frenk, C. 2007b, MNRAS, 381, 171

Miralda-Escude, J. 1995, ApJ, 438, 514

Moore, B., Governato, F., Quinn, T., Stadel, J., \& Lake, G. 1998, ApJ, 499, 5

Natarajan, P., Kneib, J.-P., Smail, I., \& Ellis, R. S. 1998, ApJ, 499, 600

Natarajan, P., Kneib, J.-P., \& Smail, I. 2002a, ApJ, 580, L11
Natarajan, P., Loeb, A., Kneib, J.-P., \& Smail, I. 2002b, ApJ, 580, L17 Navarro, J. F., Frenk, C. S., \& White, S. D. M. 1997, ApJ, 490, 493

Navarro, J. F., Hayashi, E., Power, C., et al. 2004, MNRAS, 349, 1039

Neto, A. F., Gao, L., Bett, P., et al. 2007, MNRAS, 381, 1450

Nipoti, C., Stiavelli, M., Ciotti, L., Treu, T., \& Rosati, P. 2003, MNRAS, 344, 748

Nipoti, C., Treu, T., Ciotti, L., \& Stiavelli, M. 2004, MNRAS, 355, 1119

Oke, J. B., Cohen, J. G., Carr, M., et al. 1995, PASP, 107, 375

Pointecouteau, E., Arnaud, M., \& Pratt, G. W. 2005, A\&A, 435, 1

Richard, J., Kneib, J.-P., Jullo, E., et al. 2007, ApJ, 662, 781

Richard, J., Stark, D. P., Ellis, R. S., et al. 2008, [arXiv:0803.4391]

Ricotti, M. 2003, MNRAS, 344, 1237

Sand, D. J., Treu, T., \& Ellis, R. S. 2002, ApJ, 574, L129

Sand, D. J., Treu, T., Smith, G. P., \& Ellis, R. S. 2004, ApJ, 604, 88

Sand, D. J., Treu, T., Ellis, R. S., Smith, G. P., \& Kneib, J.-P. 2008, ApJ, 674, 711

Smith, G. P., Kneib, J.-P., Ebeling, H., Czoske, O., \& Smail, I. 2001, ApJ, 552, 493

Smith, G. P., Kneib, J.-P., Smail, I., et al. 2005, MNRAS, 359, 417

Stott, J. P. 2007, Ph.D. Thesis, Durham University

Tasitsiomi, A., Kravtsov, A. V., Gottlöber, S., \& Klypin, A. A. 2004, ApJ, 607, 125

Tyson, J. A., Kochanski, G. P., \& dell'Antonio, I. P. 1998, ApJ, 498, 107

Zappacosta, L., Buote, D. A., Gastaldello, F., et al. 2006, ApJ, 650, 777 


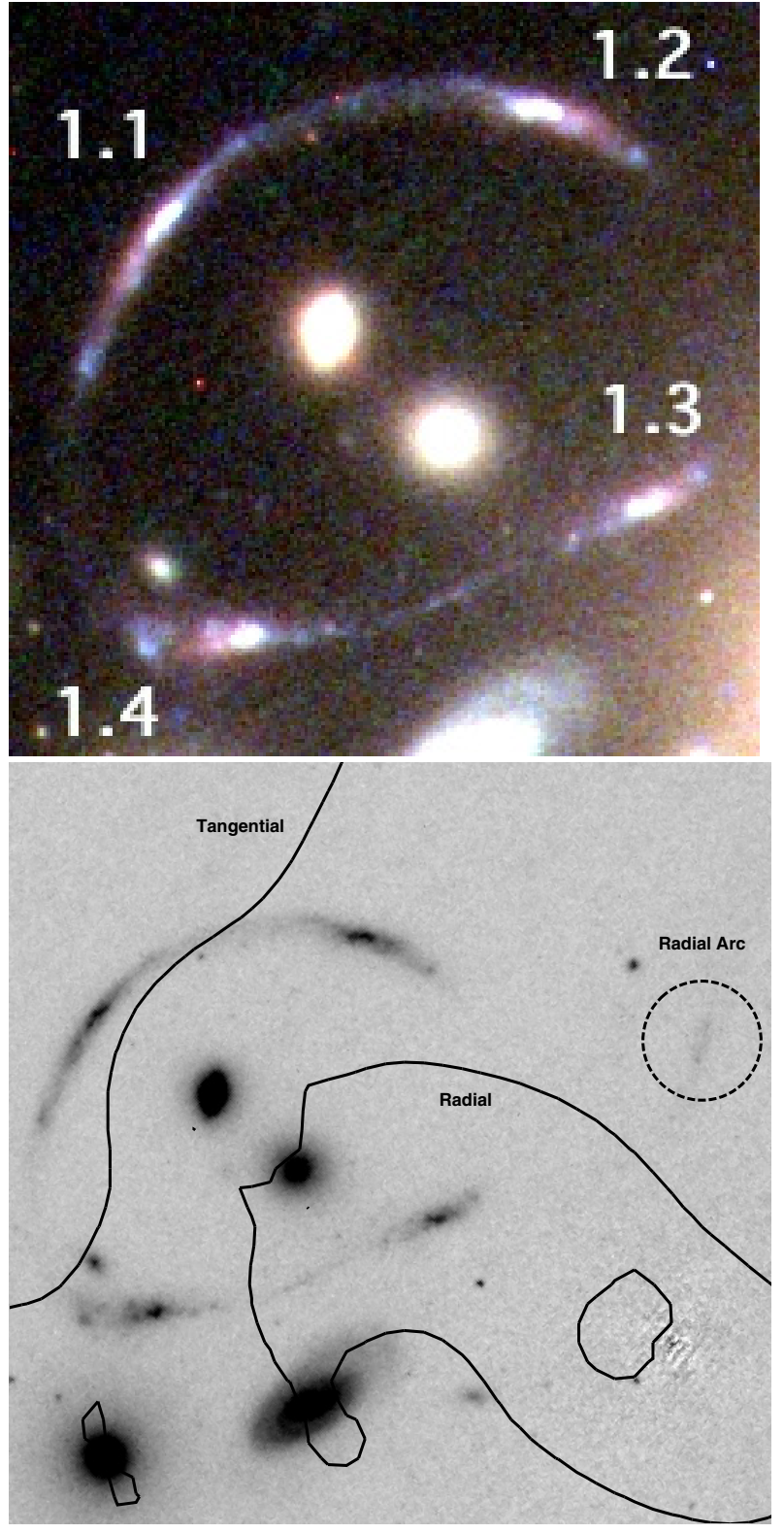

Fig. A.1. System 1, the central ring composed by 4 bright images. For its counter image, see Fig. A.8 below. Top panel: colour image from F850W, F625W and F465W observations. Size of panel is $10^{\prime \prime} \times 10^{\prime \prime}$. Bottom panel: $\mathrm{F} 775 \mathrm{~W}$ image where the light from the $\mathrm{cD}$ galaxy has been subtracted. We plot the tangential and radial critical lines at $z=$ 0.88 (redshift of system 1 ). We also report a radial feature that is coming out from the cD galaxy (see Fig. 1), that is not used in the analysis since we have not been able to detect the counter images. Size of panel is $16^{\prime \prime} \times 16^{\prime \prime}$

\section{Appendix A: Multiply imaged systems}

In this Appendix we show colour pictures of the multiply imaged systems used in this work. The size of each panel is indicated in the caption. All images are aligned with the WCS coordinates, i.e. north is Up, east is Left.

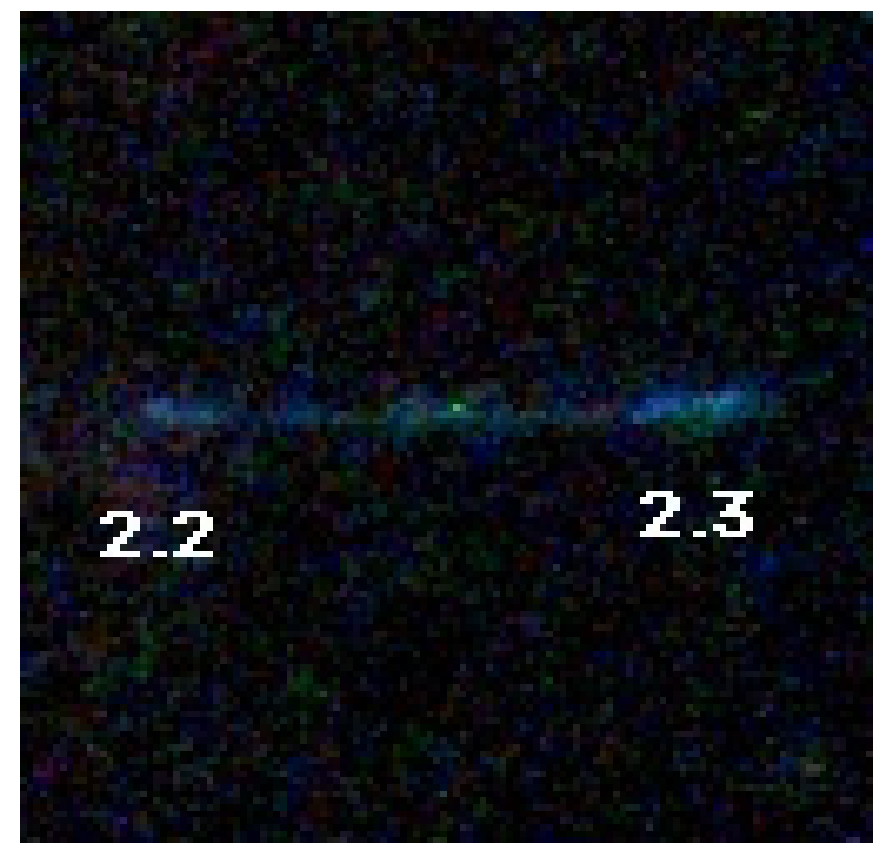

Fig. A.2. System 2, constituted by two magnified merging images. Two counter images are predicted to be more than two magnitudes fainter, we have not been able to detect any. Size of panel is $6.3^{\prime \prime} \times 6.3^{\prime \prime}$.

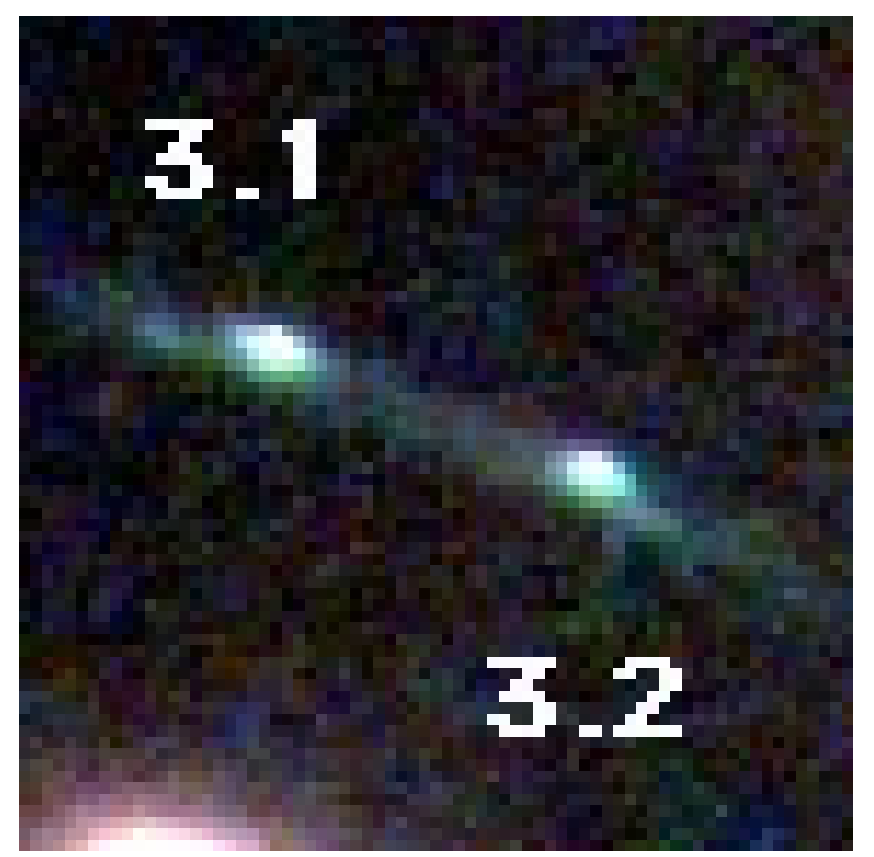

Fig. A.3. System 3, constituted by two magnified merging images and a demagnified one a bit further west. Size of panel is $4^{\prime \prime} \times 4^{\prime \prime}$. 
M. Limousin et al.: Strong lensing in Abell 1703, Online Material p 2
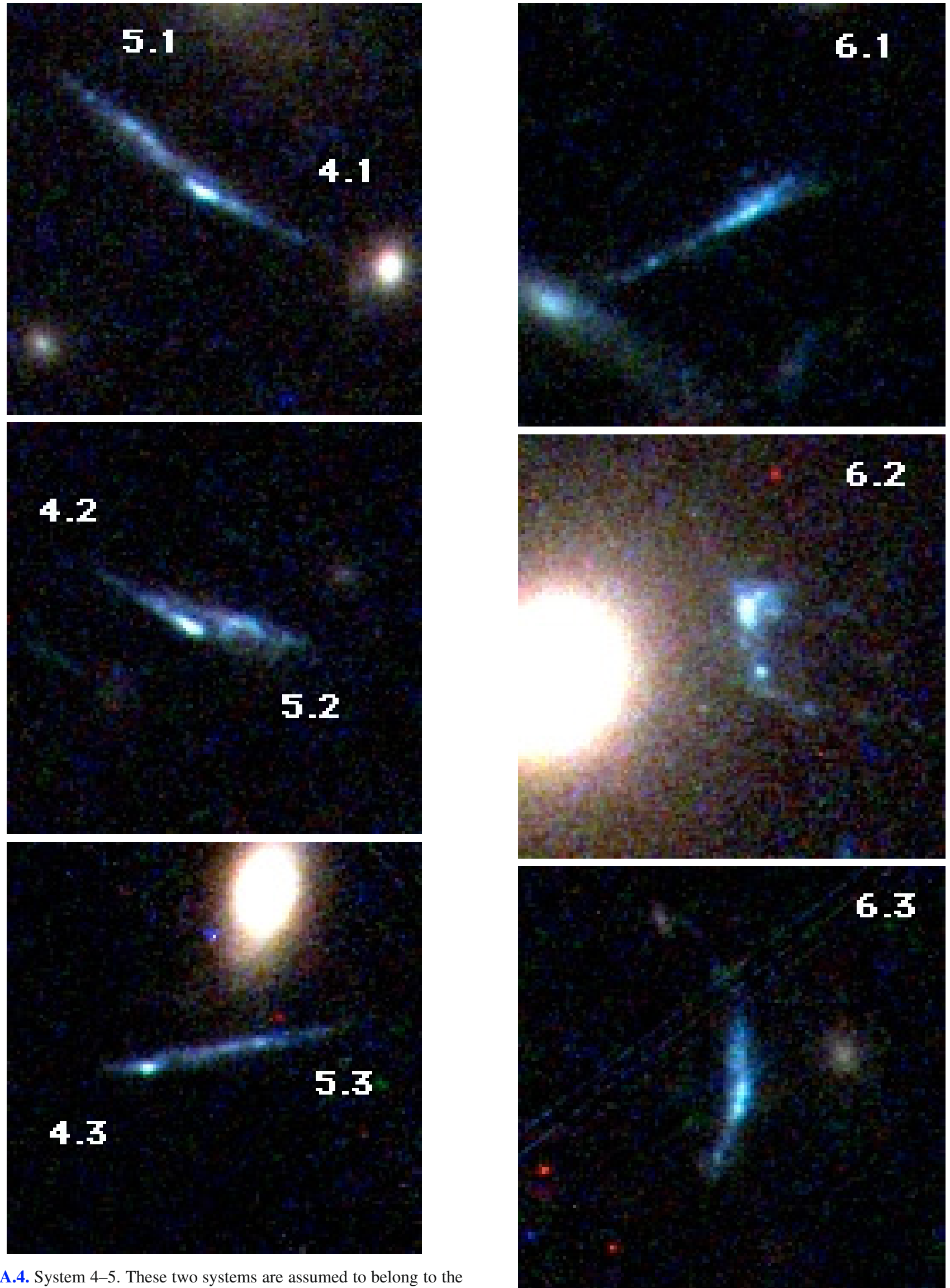

Fig. A.4. System 4-5. These two systems are assumed to belong to the same background source galaxy. System 4 is systematically brighter than system 5. From top to bottom: images 4.1 and 5.1; 4.2 and 5.2; 4.3 and 5.3. Size of each panel is $6.5^{\prime \prime} \times 6.5^{\prime \prime}$.

Fig. A.5. System 6. From top to bottom: image 6.1, 6.2 and 6.3. Size of each panel is $6.5^{\prime \prime} \times 6.5^{\prime \prime}$. 
M. Limousin et al.: Strong lensing in Abell 1703, Online Material p 3
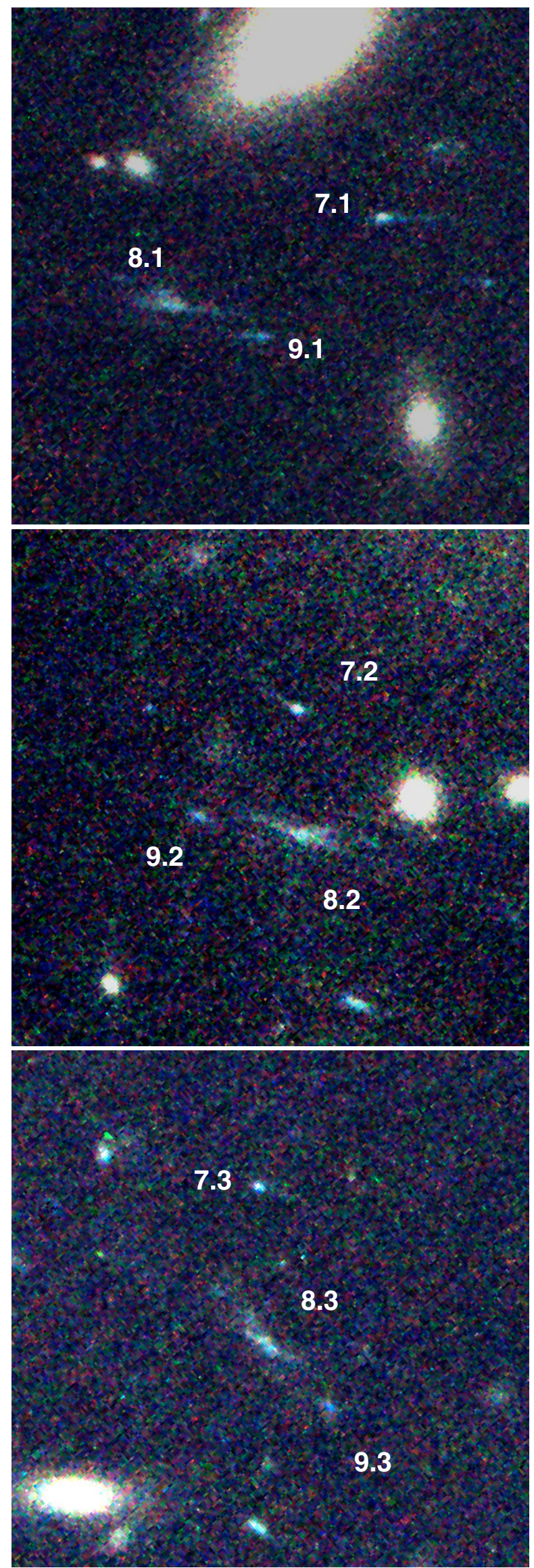

Fig. A.6. Systems 7, 8 and 9. Size of each panel is $8.5^{\prime \prime} \times 8.5^{\prime \prime}$
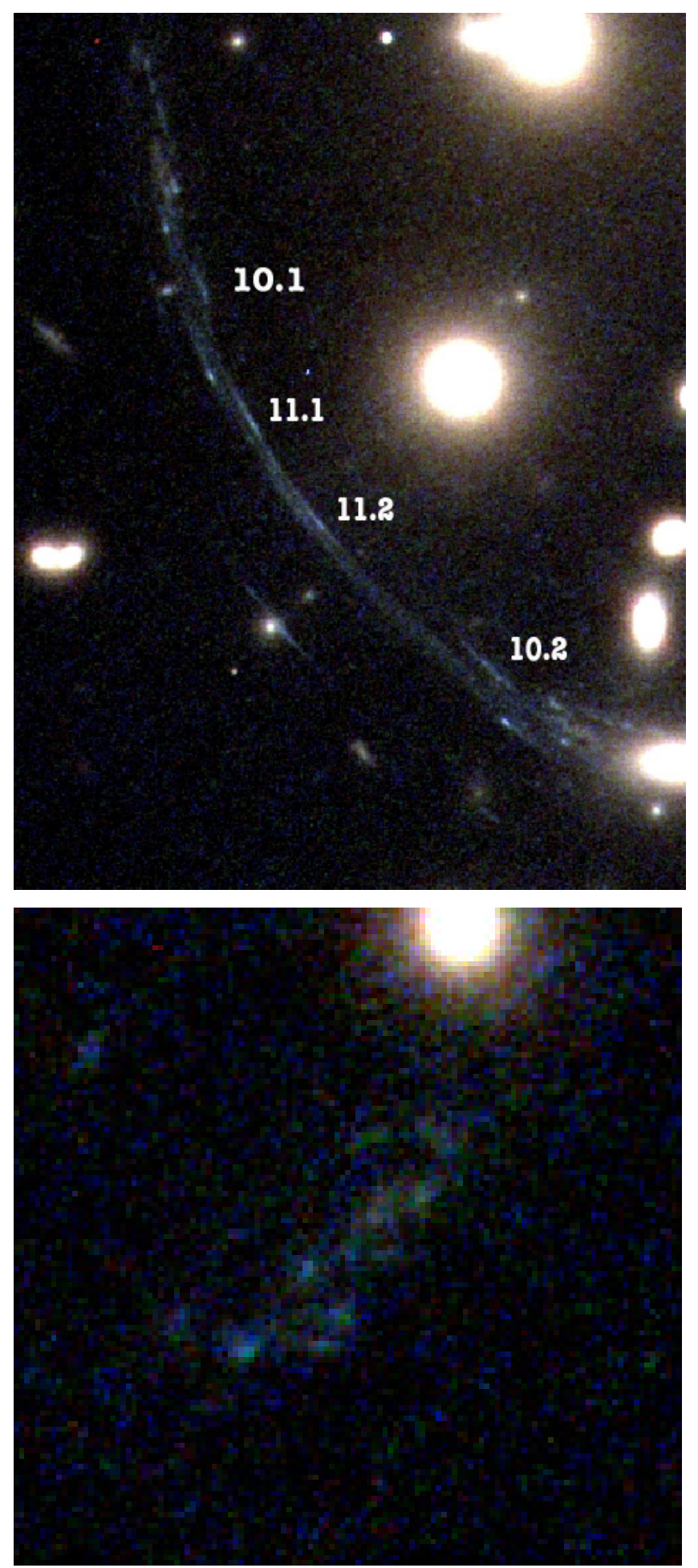

Fig. A.7. Systems 10 and 11 corresponds to two substructures identified on the giant arc. Note than more can be defined along this giant arc. Top panel: images 10.1, 11.1, 11.2 and 10.2. Size of panel is $18^{\prime \prime} \times 12^{\prime \prime}$. Bottom panel: counter image of both systems, also presenting substructures. Size of panel is $6.6^{\prime \prime} \times 6.6^{\prime \prime}$. 
M. Limousin et al.: Strong lensing in Abell 1703, Online Material p 4

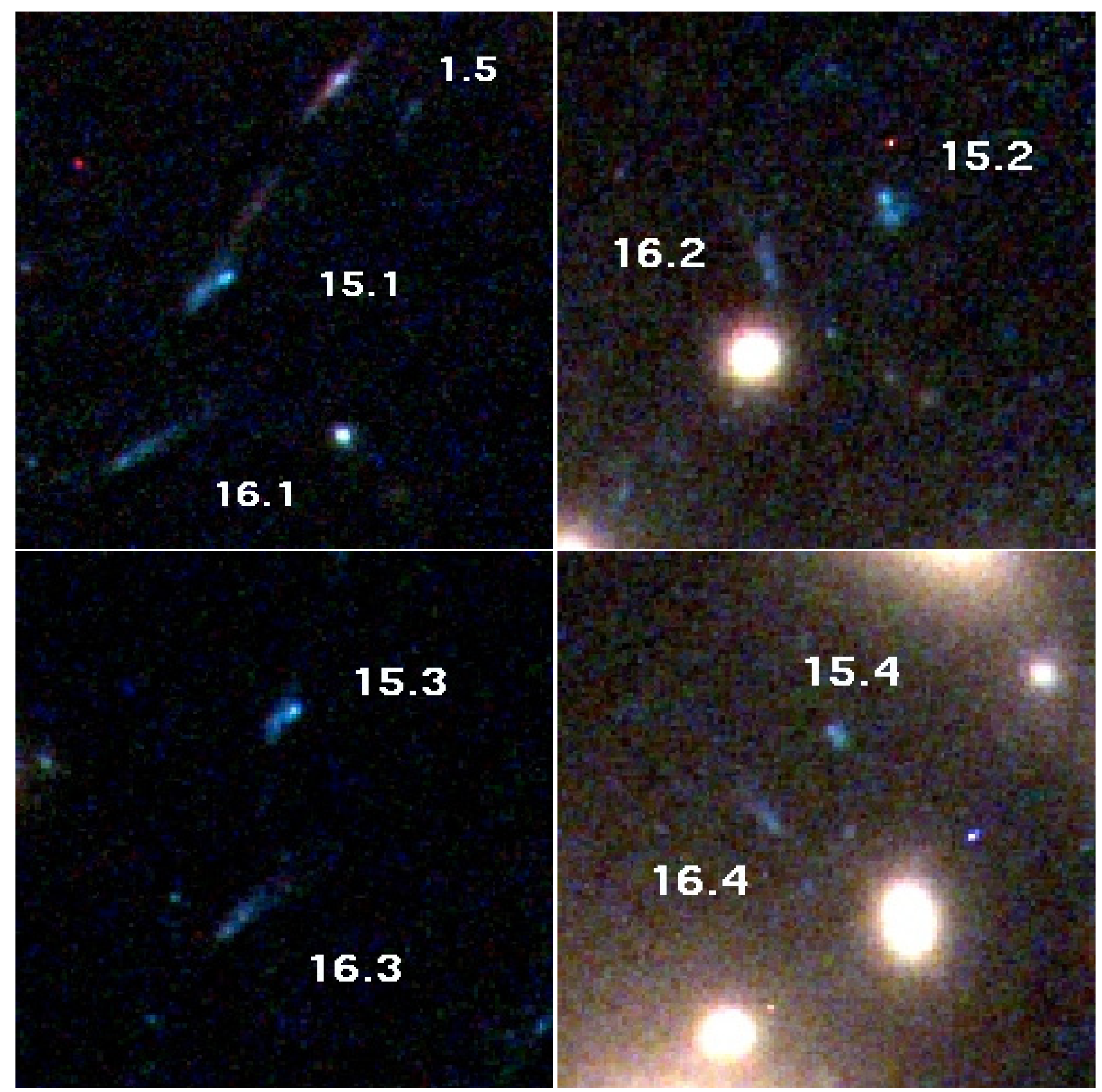

Fig. A.8. Systems 15 and 16. Images belonging to system 15 are systematically brighter than images belonging to system 16 . First panel, north to south: image 1.5, counter image of the central ring (Fig. A.1); image 15.1 and image 16.1. Second panel: images 15.2 and 16.2. Third panel: images 15.3 and 16.3. Last panel: images 15.4 and 16.4. Size of each panel is $8^{\prime \prime} \times 8^{\prime \prime}$. 
M. Limousin et al.: Strong lensing in Abell 1703, Online Material p 5

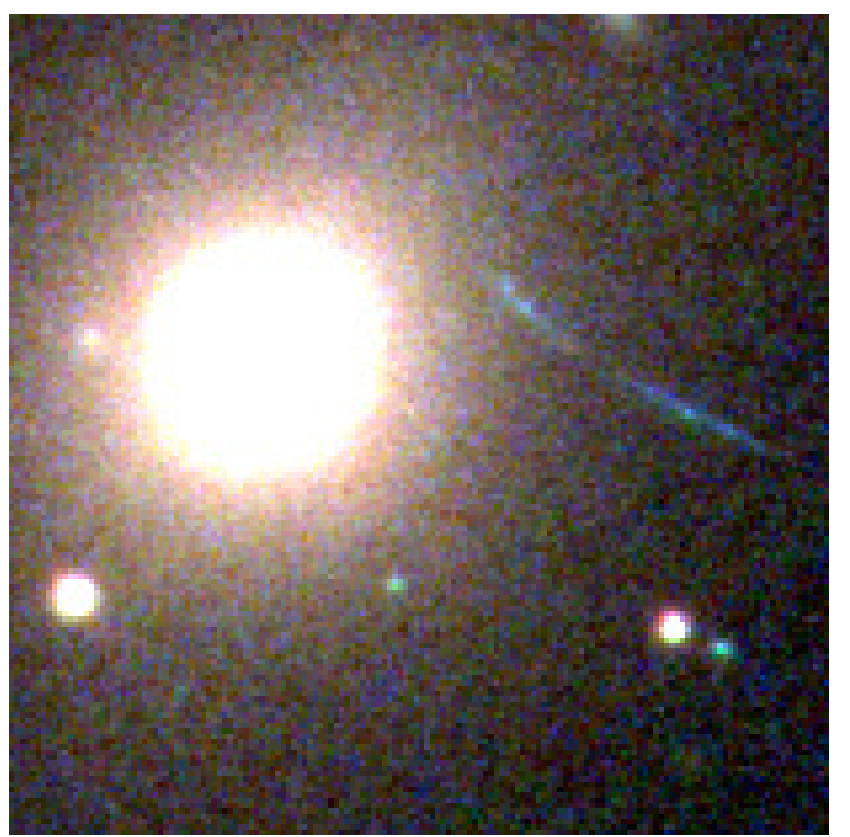

Fig. A.9. Galaxy 852, located in the northern part of the ACS field, at $\alpha=198.76342 ; \delta=51.832523$. Size of panel is $8.2^{\prime \prime} \times 8.2^{\prime \prime}$. The blue lensed feature is easily detected on this picture. 
M. Limousin et al.: Strong lensing in Abell 1703, Online Material p 6

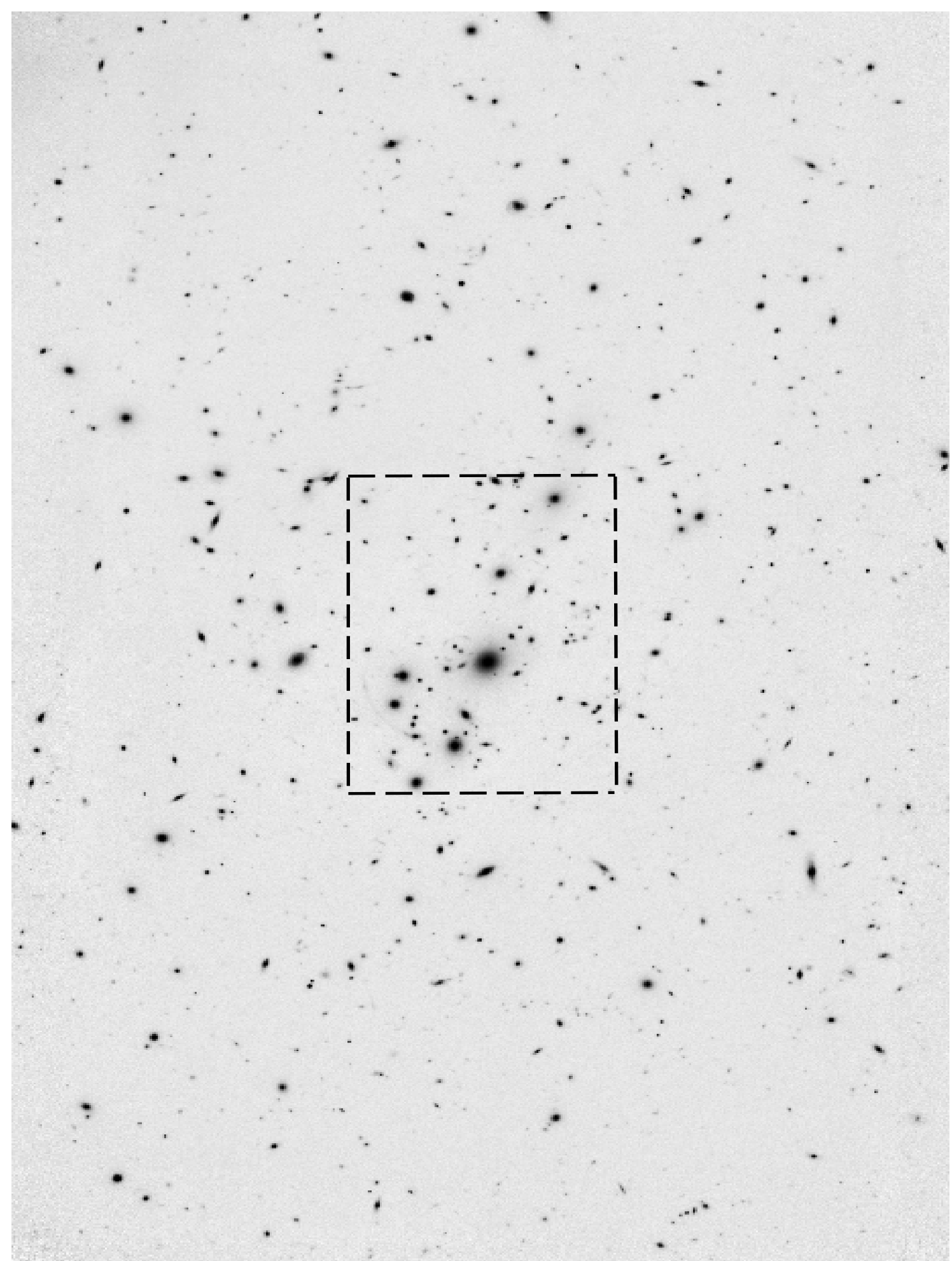

Fig. A.10. Subaru image of Abell 1703 ( $H$ band). Size of panel is $275^{\prime \prime} \times 452^{\prime \prime}$. The filamentary structure identified in Fig. 1 can be appreciated further on this image. The frame corresponds to the size of Fig. 1. 